\title{
Size-Adjustable Nano-Drug Delivery Systems for Enhanced Tumor Retention and Penetration
}

\author{
Miao Deng ${ }^{1}$ Jing-Dong Rao ${ }^{1}$ Rong Guo ${ }^{1}$ Man $\mathrm{Li}^{1}$ Qin $\mathrm{He}^{1 *}$
}

${ }^{1}$ Key Laboratory of Drug-Targeting and Drug Delivery System of the Education Ministry and Sichuan Province, Sichuan Engineering Laboratory for Plant-Sourced Drug and Sichuan Research Center for Drug Precision Industrial Technology, West China School of Pharmacy, Sichuan University, Chengdu, People's Republic of China
Address for correspondence Qin He, PhD, West China School of Pharmacy, Sichuan University, No.17, Block 3, Southern Renmin Road, Chengdu 610041, People's Republic of China

(e-mail: Qinhe317@126.com; qinhe@scu.edu.cn).

Pharmaceut Fronts 2021;3:e98-e112.

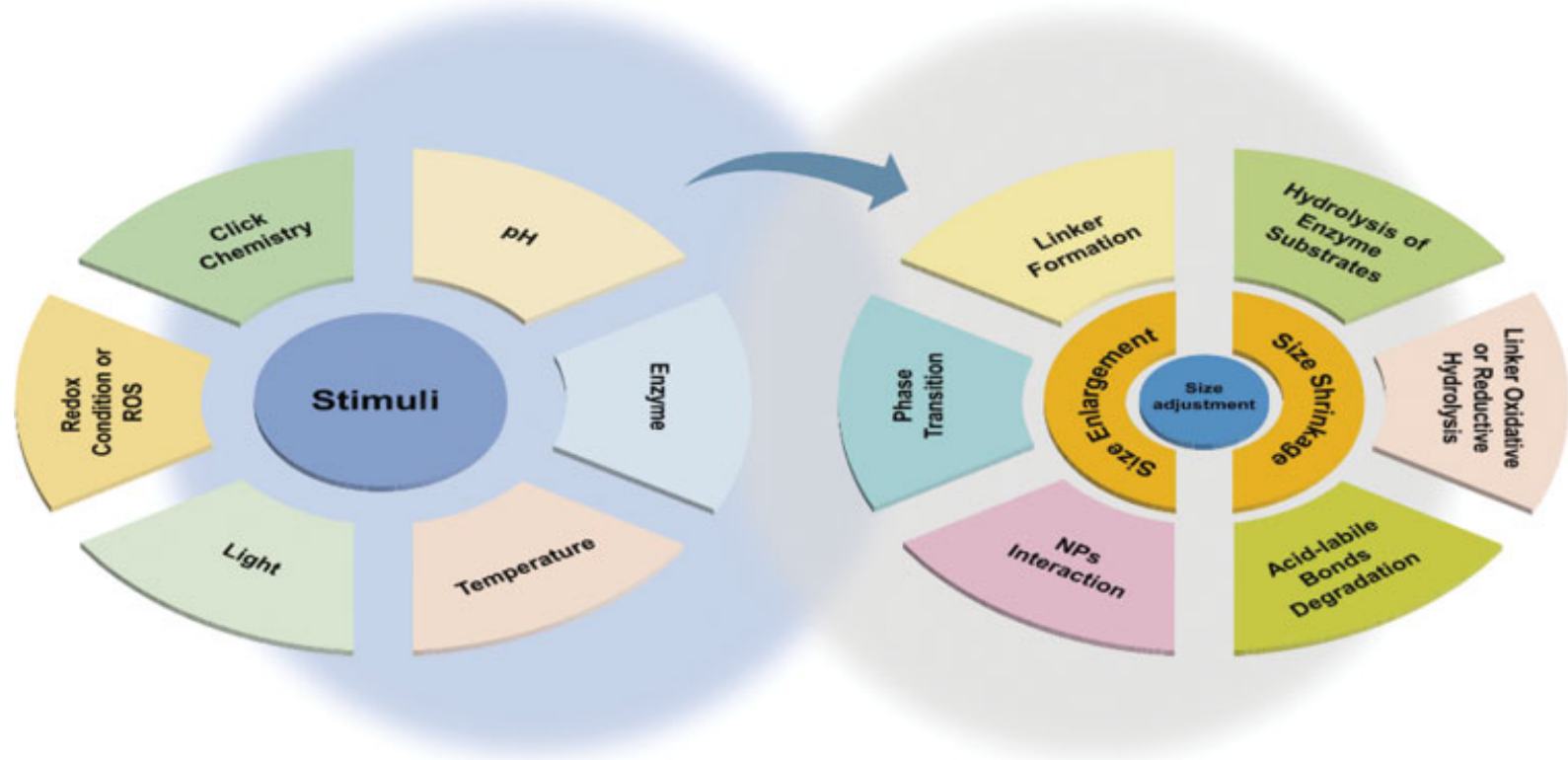

Size Adjustment Principles of Nano-Drug Delivery System
Abstract
Keywords
- size-adjustable
- nano-drug delivery systems
- stimuli-responsive
- tumor retention
- tumor penetration

Over the past decades, nano-drug delivery systems have shown great potential in improving tumor treatment. And the controllability and design flexibility of nanoparticles endow them a broad development space. The particle size is one of the most important factors affecting the potency of nano-drug delivery systems. Large-size (100-200 nm) nanoparticles are more conducive to long circulation and tumor retention, but have poor tumor penetration; small-size $(<50 \mathrm{~nm})$ nanoparticles can deeply penetrate tumor but are easily cleared. Most of the current fixed-size nanoparticles are difficult to balance the retention and penetration, while the proposal of size-adjustable nano-drug delivery systems offers a solution to this paradox. Many received

July 25, 2021

accepted

August 24, 2021
DOI https://doi.org/

$10.1055 / \mathrm{s}-0041-1736474$. ISSN 2628-5088.

\section{(c) 2021. The Author(s).}

This is an open access article published by Thieme under the terms of the Creative Commons Attribution License, permitting unrestricted use, distribution, and reproduction so long as the original work is properly cited. (https://creativecommons.org/licenses/by/4.0/)

Georg Thieme Verlag KG, Rüdigerstraße 14, 70469 Stuttgart, Germany 
endogenous and exogenous stimuli, such as acidic $\mathrm{pH}$, upregulated enzymes, temperature, light, catalysts, redox conditions, and reactive oxygen species, can trigger the in situ transformation of nanoparticles based on protonation, hydrolysis, click reaction, phase transition, photoisomerization, redox reaction, etc. In this review, we summarize the principles and applications of stimuli-responsive size-adjustable strategies, including size-enlargement strategies and size-shrinkage strategies. We also propose the challenges faced by size-adjustable nano-drug delivery systems, hoping to promote the development of this strategy.

\section{Introduction}

Tumor therapy is still one of the world-class challenges. In the past decades, nano-drug delivery systems have shown great potential in controlling drug release, reducing toxic side effects, and improving therapeutic effects. The controllability and design flexibility of nanoparticles (NPs) have attracted increasing attention in the development of precision drug delivery platforms for biomedical applications. The incomplete structure of tumor vascular endothelium provides the feasibility for the distribution of NPs to tumor sites. And the enhanced penetration and retention (EPR) effect is the main principle of NPs delivery to solid tumors. ${ }^{1}$ However, nanomedicine has not yet achieved satisfactory therapeutic effect in tumor treatment, which is mainly due to insufficient accumulation or poor permeability in tumor. ${ }^{2}$ Solid tumors possess the characteristics of high extracellular matrix (ECM) density, high interstitial fluid pressure (IFP), abnormal blood vasculature, and impaired lymphatic drainage, ${ }^{3}$ which constitute a huge obstacle for the effective accumulation and penetration of nanomedicines in tumors. Therefore, researchers are committed to adjusting the particle size, shape, surface physical and chemical properties of NPs to change their absorption, distribution, metabolism, and excretion behavior, so as to improve the therapeutic effect.

The particle size is one of the most significant factors affecting nano-drug delivery systems, including the effects of plasma clearance rate, body distribution, EPR effect, tissue diffusion, and cell internalization of NPs. ${ }^{4}$ Many studies have proved that NPs with a particle size between 30 and $200 \mathrm{~nm}$ can effectively reach the tumor site through the EPR effect, but within such a particle size range, the retention and penetration capabilities of NPs are far different. NPs with a relatively small particle size $(<50 \mathrm{~nm})$ can penetrate to the deep region of tumor but have a reduced retention due to cell efflux and backflow to the peripheral blood vessels. ${ }^{5,6}$ Contrarily, NPs with a larger particle size $(>100 \mathrm{~nm})$ have a strong retention effect in tumor, because they are easily trapped in the matrix between tumor cells and are not easy to flow back and be excreted by the cells, but in the meantime, these large particles cannot penetrate deep into the tumor. ${ }^{7,8}$ Traditional fixed-size NPs are difficult to balance accumulation and penetration. To solve this problem, researchers have proposed a series of smart size-adjustable strategies of NPs, including size-enlargement strategies and size-shrinkage strategies. These strategies are generally to achieve the initial accumulation of NPs in tumors through the EPR effect, and then transform into larger or smaller particles through endogenous or exogenous stimuli to prevent efflux or realize deep penetration.

Endogenous or exogenous stimulation is the key to realize the intelligent adjustment of NP size. Endogenous stimuli are mainly the unique characteristics of the tumor microenvironment or tumor cells, such as acidic pH, overexpressed enzymes, redox conditions, reactive oxygen species (ROS), and so on. Exogenous stimuli mainly include light, exogenous catalysts, temperature, etc. Compared with exogenous stimuli, endogenous stimuli are readily available and do not require additional equipment or reagents. Exogenous stimuli are more restrictive in application but have higher controllability. In this review, we will summarize the smart sizeadjustable strategies, including size-enlargement strategies and size-shrinkage strategies, and we will elaborate on different stimuli, such as $\mathrm{pH}$, enzyme, temperature, light, catalysts, redox, and ROS (Scheme 1). At the end, we also summarized the challenges faced by size-adjustable nanodrug delivery systems, hoping to promote the development of this strategy.

\section{Size-Enlargeable Nano-Drug Delivery System}

In fact, many NPs currently being used for biomedical applications have relatively small particle sizes, such as carbon quantum dots (QDs), magnetic NPs, gold NPs (AuNPs), and several micelles. ${ }^{9-11}$ As described above, small-sized NPs have good tumor penetration ability but are easy to be cleared, resulting in insufficient accumulation of NPs at tumor sites and limited therapeutic effect. Therefore, enhancing the retention of small-sized NPs in tumors is of great significance for achieving their functions in tumor diagnosis and treatment. Researchers have proposed a strategy to enlarge the size at tumor site by using some of the unique properties of tumor, such as acidic $\mathrm{pH}$, upregulated enzymes, to trigger the aggregation of NPs. In addition, temperature and exogenous stimuli such as light, catalysts, etc., can also be utilized to trigger the aggregation of NPs ( - Table 1 ).

\section{pH-Triggered Size Enlargement}

An acidic environment is an important feature of tumor tissue. Due to the rapid growth of tumor cells, the nutritional and metabolic environment of tumors is significantly 


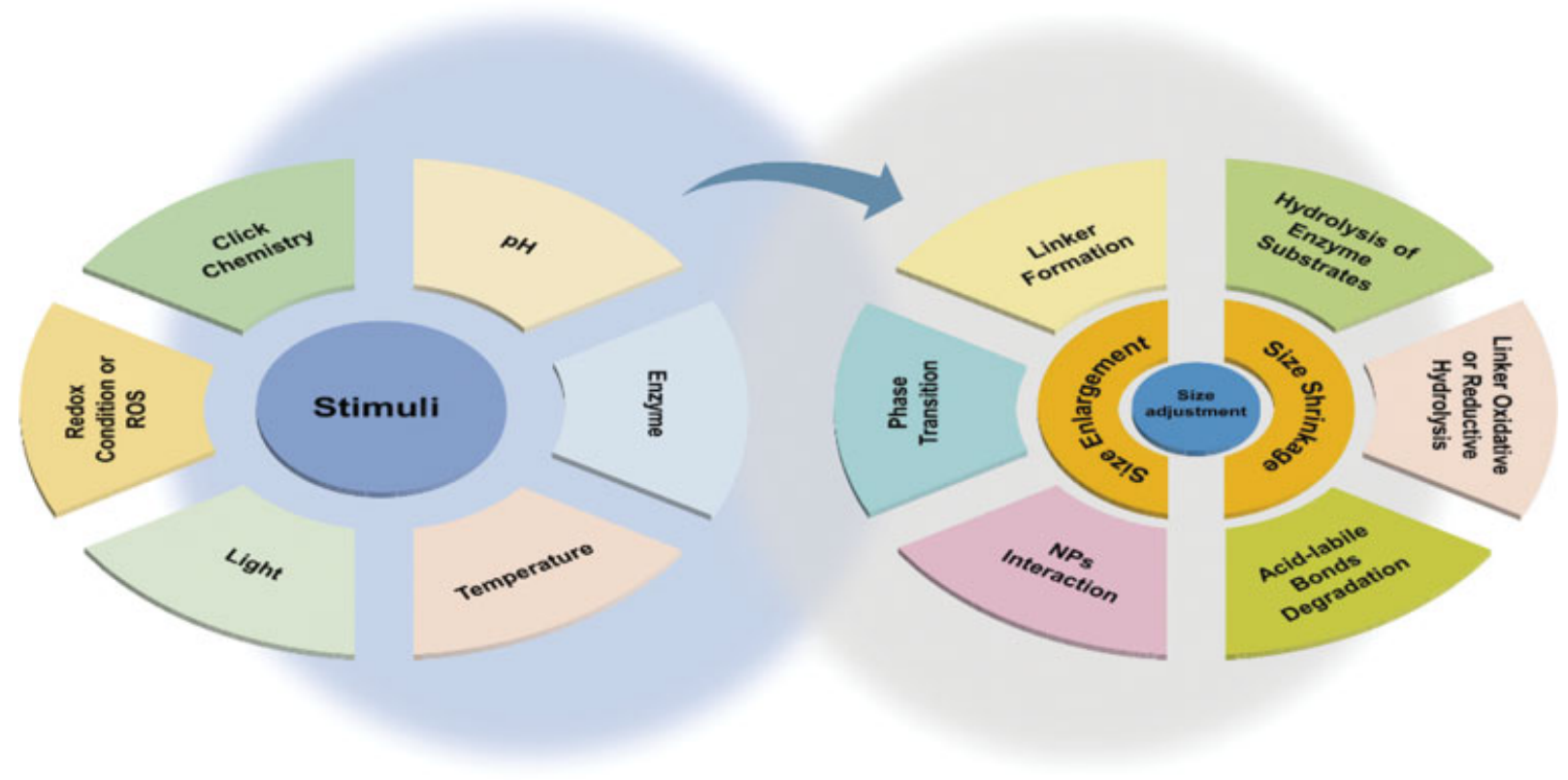

Scheme 1 Brief illustration of stimuli types and size adjustment principles of nano-drug delivery system.

Table 1 Size-enlargement strategies and typical NPs

\begin{tabular}{|c|c|c|c|c|}
\hline Stimuli & Responsive materials & Typical NPs & Size change & Ref. \\
\hline \multirow[t]{3}{*}{$\mathrm{pH}$} & Zwitterionic compounds & 16-AuNP-C10-CN4-5:5 & $\sim 50-100 \mathrm{~nm} \rightarrow \sim 100-300 \mathrm{~nm}$ & 14 \\
\hline & Block copolymer & PDPA-b-PAMA/SA@DOX·HCl & $\begin{array}{l}\sim 154 \mathrm{~nm} \rightarrow \\
\sim 1,517 \mathrm{~nm}\end{array}$ & 15 \\
\hline & Proteins & Hb-IR780 & $\begin{array}{l}\sim 6 \mathrm{~nm} \rightarrow \\
\sim 100 \mathrm{~nm}\end{array}$ & 17 \\
\hline \multirow[t]{2}{*}{ Enzyme } & $\begin{array}{l}\text { Substrates of legumain } \\
\text { (AAN, AK polypeptide, AANL, } \\
\text { AANK, etc.) }\end{array}$ & AuNPs-A\&C & $\begin{array}{l}\sim 35 \mathrm{~nm} \rightarrow \\
\sim 309 \mathrm{~nm}\end{array}$ & $7,28-30$ \\
\hline & Hyaluronic acid & CS-NG & $\begin{array}{l}\sim 123 \mathrm{~nm} \rightarrow \\
\sim 423 \mathrm{~nm}-10 \mu \mathrm{m}\end{array}$ & 34 \\
\hline Temperature & PNIPAm, PDEAm, POEGMA & PPCs & $\begin{array}{l}\sim 0 \mathrm{~nm} \rightarrow \\
\sim 600-800 \mathrm{~nm}\end{array}$ & $36-39$ \\
\hline Light & $\begin{array}{l}\text { Photosensitizers } \\
\text { (spiropyrans, azobenzene, } \\
\text { salicylideneaniline, diazirine) }\end{array}$ & dAuNPs & $\begin{array}{l}\sim 49 \mathrm{~nm} \rightarrow \\
\sim 346 \mathrm{~nm}\end{array}$ & $35,45-47$ \\
\hline $\begin{array}{l}\text { Exogenous } \\
\text { catalysts }\end{array}$ & $\begin{array}{l}\text { Click-reactive groups (azide- } \\
\text { alkyne) }\end{array}$ & M-Dox/MPLA & $\begin{array}{l}\sim 25 \mathrm{~nm} \rightarrow \\
\sim 120 \mathrm{~nm}\end{array}$ & 6 \\
\hline
\end{tabular}

Abbreviations: AAN, alanine-alanine-asparagine; AK polypeptide, alanine-alanine-asparagine-cysteine-lysine; AANL, alanine-alanine-asparagine-leucine; AANK, alanine-alanine-asparagine-lysine; NPs, nanoparticles; PNIPAm, poly(N-iso propyl acrylamide; PDEAm, poly(N-diethylacrylamide); POEGMA, poly[oligo(ethylene glycol) methacrylate].

different from that of normal tissues. ${ }^{12}$ In addition, due to the lack of oxygen in solid tumors, tumor cells produce large amounts of lactic acid through anaerobic glycolysis, resulting in low $\mathrm{pH}$ at around 6.0 to 7.0. And in some intracellular organelles such as endosomes and lysosomes, they exhibit a lower $\mathrm{pH}$ value at around 4.5 to 5.5 . While the blood circulation system and normal tissues maintain neutral $\mathrm{pH}$ of around 7.2 to $7.4 .^{13}$ Researchers utilized the differences in $\mathrm{pH}$ as a stimulus to design a variety of size-adjustable nano-drug delivery systems.
pH-sensitive materials are usually zwitterionic compounds, and size enlargement is usually attributed to the breakdown of the charge balance of the materials in an acidic environment, NPs aggregate by electrostatic attraction, van der Waals attraction, hydrogen-bonding attraction, or other forces. Liu et al modified AuNPs with mixed self-assembled monolayers of weak electrolytic 11-mercaptoundecanoic acid (HS-C10-C) and strong electrolytic (10-mercaptodecyl) trimethylammonium bromide (HSC10-N4) to obtain pH-sensitive mixed-charge zwitterionic 


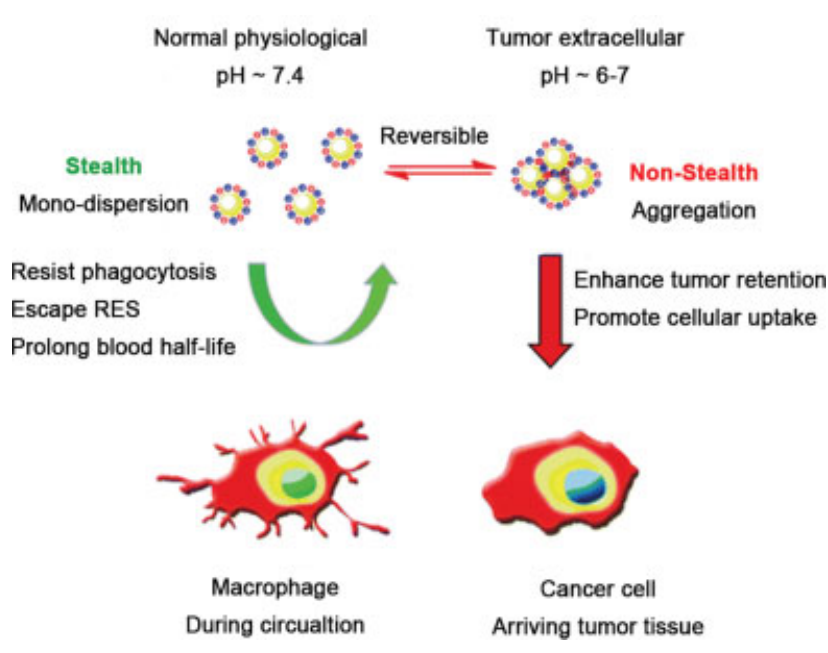

Fig. 1 Schematic illustration of the targeting of acidic tumor microenvironment by $\mathrm{pH}$-responsive mixed-charge zwitterionic AuNPs (symbols on the NP [yellow color] surface mean positively charged groups [blue color] and negatively charged groups [red color] localized on the NP surface simultaneously). During circulation in the blood, the NPs with small size and the zwitterionic surface exhibit a prolonged circulation time and hence can leak into tumor sites via the EPR effect. Arriving at the tumor site, stimulated by the tumor extracellular $\mathrm{pH}$ ( $\mathrm{pHe}$ ), the zwitterionic AuNPs form aggregates and sedimentate in the tumor space, leading to enhanced retention and cellular uptake of NPs in the tumor. Copyright 2013 American Chemical Society. AuNPs, gold NPs; EPR, enhanced penetration and retention; NPs, nanoparticles.

AuNPs. ${ }^{14}$ At high $\mathrm{pH}$, the zwitterionic AuNPs remained stable due to strong hydration and electrostatic repulsion, as the $\mathrm{pH}$ decreased to 7.0 to 5.5 , the HS-C10-C ligands were partially protonated, resulting in weaker hydration and increased hydrogen bond attraction. When the van der Waals force plus the hydrogen-bonding attraction exceeded the hydration and electrostatic repulsions, NP aggregation occurred. When the $\mathrm{pH}$ was lower than 5.5 , NPs dispersed again due to the strong electrostatic repulsions of positive quaternary ammonium (-Fig. 1). In another case, $\mathrm{Wu}$ et al reported a $\mathrm{pH}$-sensitive block copolymer succinic anhydride (SA)-modified poly(2-diisopropylaminoethyl methacrylate)-block-poly(2-aminoethyl methacrylate hydrochloride) (PDPA- $b$-PAMA/SA), ${ }^{15}$ where the PDPA block could be protonated under a slightly acidic environment to be positively charged, and then combined with negatively charged PAMA/SA through electrical interaction to form NP aggregation.

In addition, proteins with acidic or basic amino acid residues are regarded as natural $\mathrm{pH}$-sensitive nanoplatforms. Proteins exhibit unique colloidal stability at different $\mathrm{pH}$ values, while aggregate near the isoelectric point $(\mathrm{pI}){ }^{16}$ Thus, proteins with $\mathrm{pI}$ close to the slightly acidic $\mathrm{pH}$ of tumors can be used as a tumor-responsive carrier to enhance tumor retention. Li et al utilized bovine hemoglobin as the $\mathrm{pH}$-sensitive nanocarrier of the near-infrared (NIR) dye IR780 iodide to prepare the Hb-IR780 complexes, which could be well dispersed at the $\mathrm{pH}$ of normal tissues, while aggregated in the acidic tumor environment. ${ }^{17}$

\section{Enzyme-Induced Size Enlargement}

Enzymes play a vital role in the process of biological metabolism, and enzymes produced by cells participate in various physiological activities. ${ }^{18}$ In the process of tumor development and invasion, the regulation of some enzymes will support tumor growth and lay the foundation for tumor pathology. ${ }^{19}$ The expression of these enzymes has become a sign of tumor tissues and an important target for drug delivery. Due to the high selectivity between enzymes and substrates, the catalysis of enzymes possesses high specificity and can significantly reduce off-target effects. Therefore, many enzymes are used to trigger the release of drugs or achieve effective tumor targeting. There are many specific overexpressed enzymes in tumor tissues including legumain, ${ }^{20}$ hyaluronidase (HAase), ${ }^{21}$ matrix metalloproteinase (MMP), ${ }^{22}$ cathepsin $B,{ }^{23}$ furin, ${ }^{24}$ phospholipase $A 2,{ }^{25}$ etc.

Legumain is a highly conserved aspartate endonuclease with highly stringent position specificity for cleavage and hydrolysis, which is found to be overexpressed in a variety of solid tumors and is positively correlated with the aggressiveness and metastasis of malignant tumors. ${ }^{26,27}$ Therefore, as an enzymatic target, legumain has potential for prodrug activation and responsive delivery of nano-drug systems. In the development of drug carriers, the substrates of legumain are usually introduced, which are generally polypeptides containing aspartate such as alanine-alanine-asparagine $(\mathrm{AAN}),{ }^{28}$ alanine-alanine-asparagine-cysteine-lysine

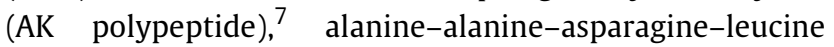
(AANL), ${ }^{29}$ alanine-alanine-asparagine-lysine (AANK), ${ }^{30}$ etc. Ruan et al constructed a legumain-responsive AuNP drug delivery system AuNPs-A\&C, ${ }^{7}$ which consisted of AK polypeptide-modified AuNPs (AuNPs-AK) and 2-cyano-6aminobenzothiazole-modified AuNPs (AuNPs-CABT). After entering the blood circulation, AuNPs-A\&C reached the tumor site through the EPR effect. Then the AK polypeptide of AuNPs-AK was hydrolyzed by the overexpressed legumain in the tumor to expose the 1,2-thiolamino groups of cysteine, which further click-reacted with the cyano group of CABT, leading to the aggregation of two AuNPs and enhanced tumor retention of the AuNPs (-Fig. 2A).

Hyaluronic acid (HA) is a recognized ligand for the cell surface receptors such as CD44 and can be degraded by HAase. ${ }^{31,32}$ Notably, both CD44 and HAase are highly related to the progression of cancer and overexpressed in tumor. ${ }^{33}$ Thus, HA is usually introduced into nano-drug delivery systems as a ligand for actively targeting tumors or as a tumor-responsive material. $\mathrm{Hu}$ et al utilized the highly expressed HAase in the tumor microenvironment to construct a nanocarrier (designated CS-NG) that can aggregate outside the cell to form extracellular depots. ${ }^{34} \mathrm{CS}-\mathrm{NG}$ was a core-shell-based nanogel. The core was an acid-sensitive nanogel loaded with tumor necrosis factor-related apoptosis inducing ligand (TRAIL) and antiangiogenic cilengitide. The shell loaded with exogenous catalysts transglutaminase (TG) was formed by the interfacial polymerization of HA and further decorated with human serum albumin (HSA). After intravenous administration, CS-NG accumulates at the tumor site through the EPR effect and the targeting effect 
A
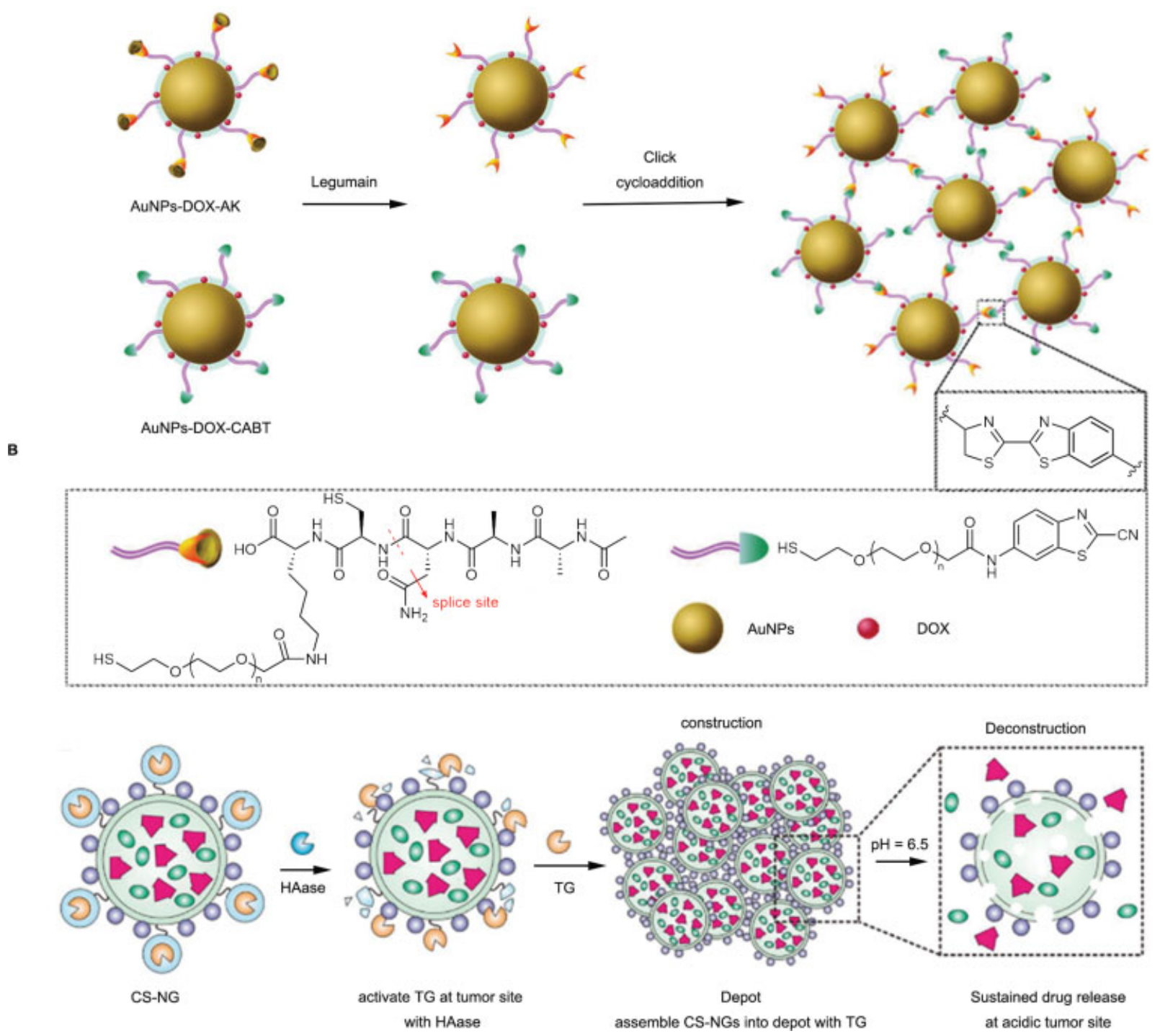

Fig. 2 (A) Diagram depicting the legumain-triggered aggregation and composition of AuNPs-DOX-A\&C. The AuNPs-DOX-AK undergoes a cleavage by legumain to expose the 1,2-thiolamino group of cysteine, where click cycloaddition further occurs with the contiguous cyano group of AuNPs-DOX-CABT to form aggregates. Copyright 2016 American Chemical Society. (B) A typical CS-NG is consisted of core-NG (C-NG) loaded with therapeutics, shell-NG (S-NG) with encapsulated transglutaminase (TG), and human serum albumin (HSA), coated on the surface of C-NG. The polymeric matrix of C-NG is acid-degradable, and the polymeric matrix of S-NG is made from hyaluronic acid (HA) that can be degraded by HAase. At the tumor site, the overexpressed HAase digests the HA and releases TG, which catalyzes the cross-linking of HSA and assembles CS-NG into the "drug-delivery depots." The matrix of C-NG can be gradually degraded and subsequently release the encapsulated therapeutics at the acidic tumor microenvironment. Copyright 2016 American Chemical Society.

mediated by the interaction of HA and CD44 receptors. The overexpressed HAase in the tumor microenvironment could degrade the HA shell, resulting in the exposure of TG, which catalyze the reaction between the amine group on the surface of the core nanogel and the acyl group on HSA to form peptide bonds, so that the nanogel was cross-linked to form large-size depots ( $\mathbf{- F i g . 2 B}$ ). These depots could inhibit cellular internalization and prolong tumor retention, thereby enhancing cytotoxicity and improving antitumor efficacy.

\section{Temperature-Responsive Size Enlargement}

Polymer solutions usually possess both a lower critical solution temperature (LCST) and an upper critical solution temperature. At a temperature lower than the LCST, the polymer can be completely miscible in the solvent in all proportions, while at a temperature higher than the LCST, the polymer will undergo a phase transition and precipitates from the solvent. ${ }^{35}$ Many natural and synthetic polymers possess temperature responsiveness, such as poly $(N$-iso propyl acrylamide) (PNIPAm), ${ }^{36}$ poly( $N$-diethylacrylamide) (PDEAm), ${ }^{37}$ and poly[oligo(ethylene glycol) methacrylate] (POEGMA). ${ }^{38}$ Notably, the LCST of the polymer can be controlled by adjusting the hydrophilicity, grafting group, and chain length, which provides possibilities for the development of thermal-responsive NPs. ${ }^{35}$ For instance, Qiao et al constructed a kind of polymeric peptide conjugates (PPCs) 


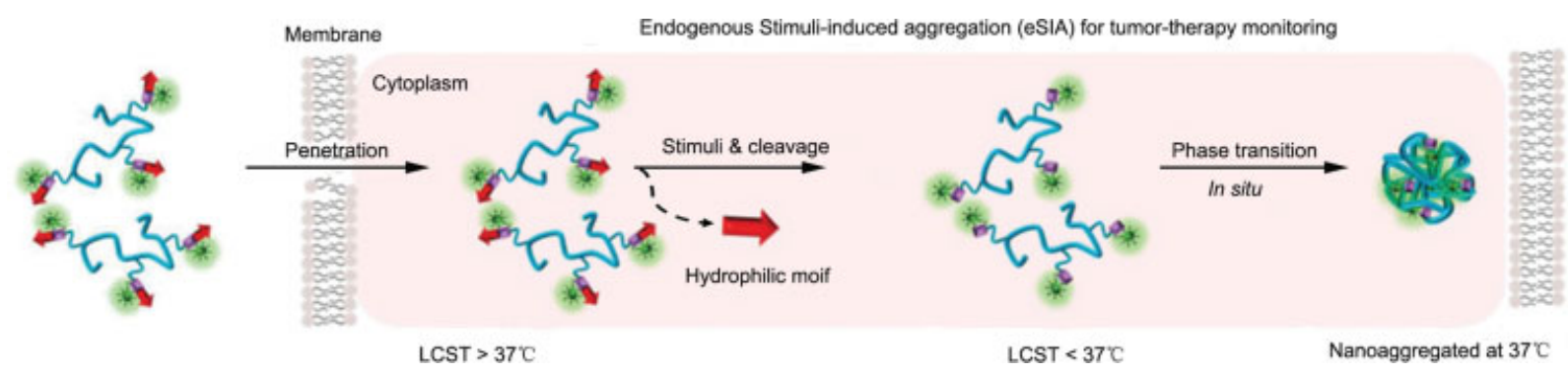

Fig. 3 Schematic representation of stimuli-instructed construction of controllable nanoaggregates for monitoring tumor therapy response. Copyright 2017 American Chemical Society.

that could self-assemble and aggregate in cells. ${ }^{39}$ PPCs consist of three parts, a thermal-responsive polymer backbone, a grafted peptide, and a signal molecule. The LCST of PPCs is over $37^{\circ} \mathrm{C}$, which ensures the stability of PPCs. After being internalized by cells, the grafted ligand on the polymer chain will be cleaved by intracellular enzymes, resulting in a decrease in LCST to less than $37^{\circ} \mathrm{C}$, and then the phase transition occurs to form aggregates with a size of 600 to 700 nm (-Fig. 3). This temperature-triggered transformation prolongs the residence of PPCs in cells, which is helpful for monitoring tumor cell apoptosis and tumor treatment response.

In addition, photothermal molecules and exogenous light are often introduced into nano-drug delivery systems to achieve response to different temperatures. For example, Liu et al synthesized PPCs integrating a poly( $\beta$-thioester) as a thermal-responsive backbone, a functional peptide (cytotoxic peptide and cell-penetrating peptide), and NIR photothermal molecules. ${ }^{40}$ The initial PPCs with small size $(<10 \mathrm{~nm})$ could penetrate deeply into the tumor at body temperature, while under the irradiation of NIR laser, the temperature raised and led to the intratumoral self-assembly of PPCs with the size increasing to 30 to $40 \mathrm{~nm}$, and thus enhanced tumor accumulation and cellular internalization.

\section{Light-Sensitive Size Enlargement}

Light-mediated drug delivery has received extensive attention in tumor treatment due to its noninvasiveness, spatiotemporal addressability, and high therapeutic efficiency. ${ }^{41}$ Light, including ultraviolet (UV) light, visible light, blue light, NIR light, etc., can serve as a phototherapy or photothermal therapy agent to directly kill tumors, and can also be used as a stimulus to precisely control drug release and trigger the transformation of NPs. ${ }^{42-44}$ To achieve light-triggered selfassembly of NPs, polymers are usually conjugated with lightsensitive molecules (photosensitizers) such as spiropyrans, ${ }^{45}$ azobenzene, ${ }^{46}$ salicylideneaniline, ${ }^{35}$ etc. These photosensitizers can undergo isomerization or dimerization at a specific wavelength to form covalent bonds, leading to the crosslinking of NPs. For example, Cheng et al reported a photocross-linkable AuNP based on photo-labile diazirine. ${ }^{47}$ Under $405 \mathrm{~nm}$ laser irradiation, the diazirine groups on the surface of NPs transformed into reactive carbene, which then formed covalent bonds with ligands of adjacent AuNPs and lead to the formation of cross-linked aggregates. The aggregated
AuNPs further facilitated photothermal treatment with NIR light (-Fig. 4).

However, what is worth noting is that the penetration ability of UV light, visible light, and blue light in biological tissues is weak, thus limiting their in vivo application. While NIR light exhibits stronger tissue penetration because most tissues and biological fluids can absorb a wavelength range of 700 to $1,100 \mathrm{~nm}^{48}$ If the penetrating NIR light can be converted into UV-visible light or blue light, the application of photosensitizers will be greatly expanded. It is reported that rare-earth metals such as lanthanide ions $\left(\mathrm{Yb}^{3+}, \mathrm{Er}^{3+}\right.$, and $\mathrm{Tm}^{3+}$ ) have the ability to convert NIR light into shortwavelength light via excitation by two or more photons. ${ }^{49}$ And NPs containing these rare-earth metals, also called upconversion NPs (UCNPs), have received great attention. ${ }^{48}$ However, light-sensitive size enlargements based on UCNPs are rarely reported. Zhao et al demonstrated that NIR-treated UCNPs could emitted UV/visible photons and triggered the photoisomerization of azobenzene, ${ }^{50}$ which is commonly used for light-sensitive aggregation; therefore, UCNPs have great potential in light-sensitive aggregation and deserve further investigation.

\section{Click-Chemistry-Mediated Size Enlargement}

Click chemistry has been widely used in chemical synthesis and biological systems due to its high efficiency, high selectivity, and simple and benign proceeding conditions. ${ }^{51}$ Click reactions include azide-alkyne cycloaddition, thiol-ene, oxime, Diels-Alder, Michael addition, and pyridyl sulfide reactions. $^{52}$ Among them, copper-catalyzed azide-alkyne cycloaddition (CuAAC) is by far the most used click reaction. To achieve size enlargement of NPs, click-reaction groups are usually modified on the surface of NPs, and exogenous catalysts or other stimulus are combined to promote the interaction of groups to form covalent crosslinks. Our group modified the azide or alkyne group on DSPE-PEG micelles $(\sim 25 \mathrm{~nm})$, after the micelles reach tumor tissues, copper sulfate and sodium ascorbate were intratumorally injected to catalyze the cycloaddition between azide and alkyne groups, leading to an increase in NP size and enhanced tumor retention and accumulation of NPs ( $\mathbf{- F i g . 5 A})^{6}$

However, it is undeniable that intratumor injection of catalyst increased the burden on objects and $\mathrm{Cu}(\mathrm{I})$ might cause biotoxicity. In recent years, a more biocompatible copper-free click chemistry based on strain-promoted 

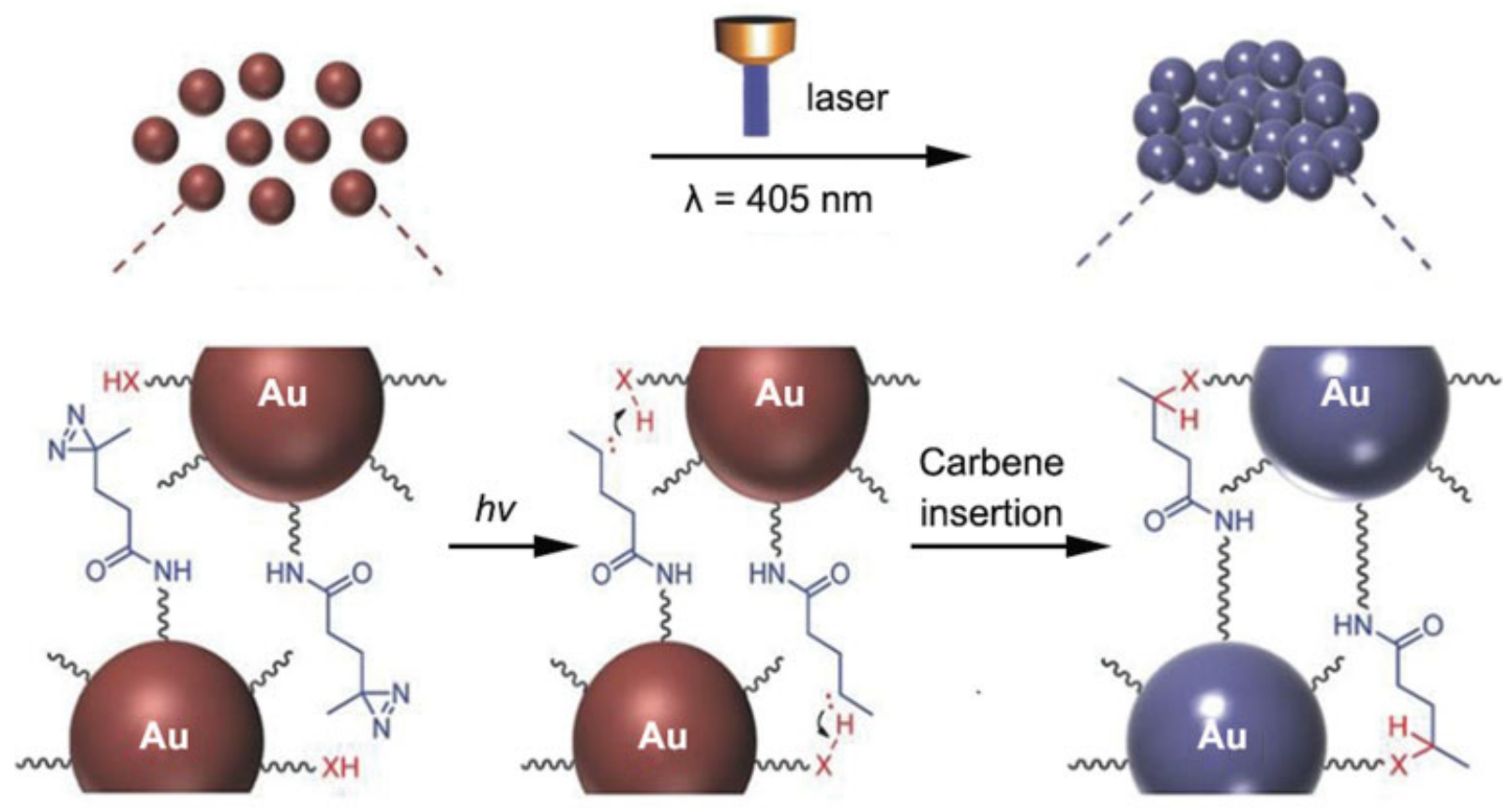

$$
\mathrm{X}=\mathrm{C}, \mathrm{N}, \mathrm{O}, \mathrm{S}
$$

Fig. 4 Schematic illustration of light-triggered assembly of dAuNPs. Copyright 2016 WILEY-VCH Verlag GmbH \& Co., KGaA, Weinheim.

alkyne-azide cycloaddition (spAAc), which does not require a metal catalyst, has evolved and utilized in tumor-targeted drug delivery systems. ${ }^{53}$ The most commonly used molecular pairs with copper-free click reactivity include azide and dibenzylcyclooctyne (DBCO), ${ }^{54}$ azide and bicyclo[6.1.0.]nonyne $(\mathrm{BCN}),{ }^{55}$ tetrazine $(\mathrm{Tz})$, and trans-cyclooctenes (TCO). ${ }^{56}$ Our group utilized azide and DBCO to construct a sizeenlargeable nanoplatform by modifying them on polycaprolactone-polyethylene glycol (PCL-PEG 2000 ) micelles, which were further coated with acid-cleavable PEG $_{5000}$. This nanoplatform could respond to acidic tumor microenvironment and intracellular lysosomes, causing the shedding of PEG coating and the exposure of azide and DBCO, followed by the copper-free click reaction and the aggregation of micelles (-Fig. 5B). ${ }^{57}$

\section{Size-Shrinkable Nano-Drug Delivery System}

Some tumors, such as pancreatic tumor and breast tumor, have dense ECM and high fluid interstitial pressure. NPs with large size can hardly pass through the peripheral matrix barrier to reach the deep region of tumor. In addition, to treat some multidrug resistant cells, it is necessary to deliver drugs into the nucleus, while the small size of the nuclear pore $(\sim 10-39 \mathrm{~nm})$ greatly limits the entry of NPs. ${ }^{58}$ Therefore, the size-shrinkage strategy of NPs is of great significance in dense tumor and nuclear-targeted drug delivery. Many studies have proposed that acidic environment, extracellular upregulated enzymes, proteases bound to the cell surface, redox environment, and ROS can trigger the extracellular and intracellular size shrinkage of NPs (-Table 2 ).

\section{pH-Triggered Size Shrinkage}

Size shrinkage triggered by $\mathrm{pH}$ is usually based on acid-labile chemical bonds and pH-responsive polymers. Acid-labile chemical bonds, including acetal, orthoester, hydrazone, imine, oxime, and cis-aconyl bonds, are stable at neutral $\mathrm{pH}$ but will undergo degradation or hydrolysis in an acidic environment, causing the nano-system to disintegrate to release drugs or shrink in size. ${ }^{59}$ Their chemical structures and hydrolyzed products are shown in - Table $3 .^{60-65}$ Due to the proximity of the pendent carboxylic acid, cis-aconyl bonds could undergo intramolecularly assisted C-4 acid-catalyzed hydrolysis at the C-1 bond. Li et al constructed a size-shrinkable nano system iCluster/Pt, which was prepared from coassembly of platinum (Pt) prodrug-conjugated poly(amidoamine)-graft-polycaprolactone (PCL-CDM-PAMAM/Pt), poly(ethylene glycol)- $b$-poly ( $\varepsilon$-caprolactone) (PEG- $b$-PCL), and PCL homopolymer by nanoprecipitation method. ${ }^{66}$ In the PCL-CDM-PAMAM/Pt part, Ptconjugated PAMAM was coupled to PCL through a cis-aconyl bond, in particular, PCL was first reacted with 2-propionic-3methylmaleic anhydride (CDM) to produce PCL-CDM, then Ptconjugated PAMAM was coupled to PCL-CDM through the reaction of the amino groups of PAMAM with the CDM anhydride residue. At physiological $\mathrm{pH}$, the iCluster/Pt has an initial size of around $100 \mathrm{~nm}$, which is favorable for long blood circulation and enhanced tumor accumulation through the EPR effect. Once in the acidic tumor environment, the acid- 

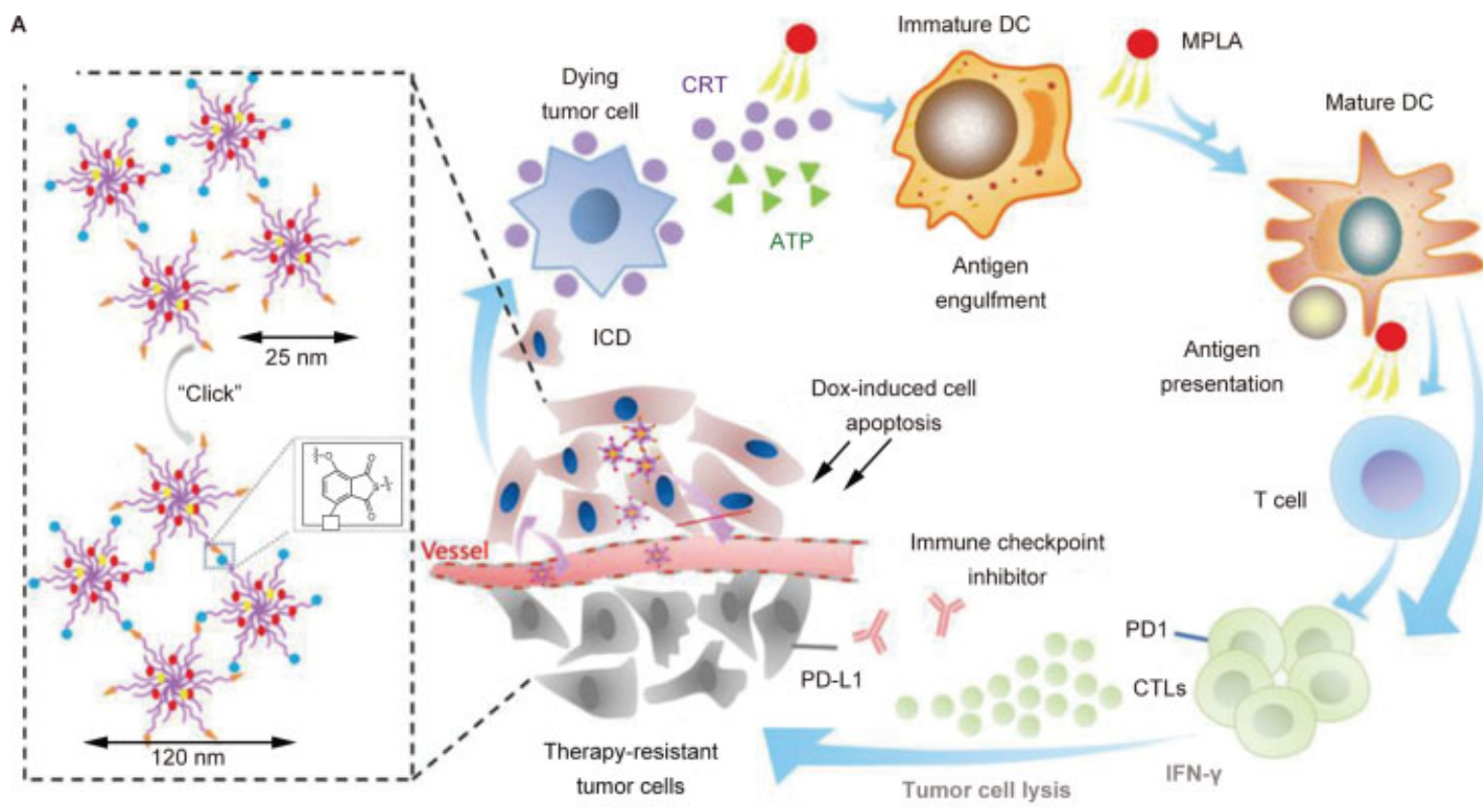

Dox-induced cell apoptosis
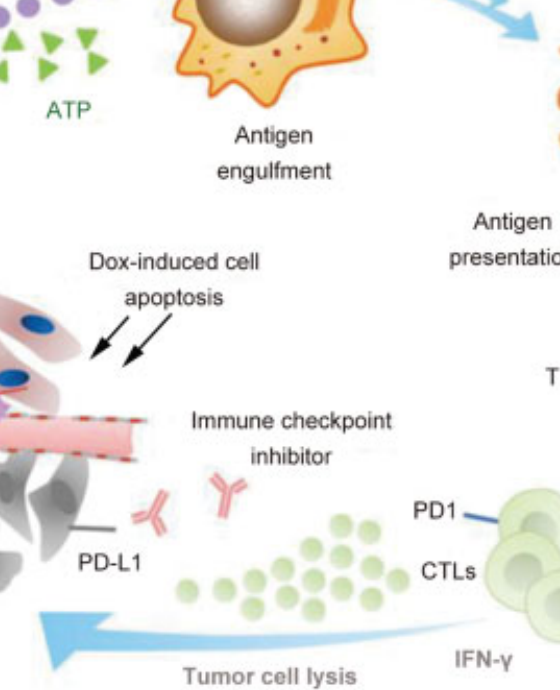

Antigen presentation T cell

B

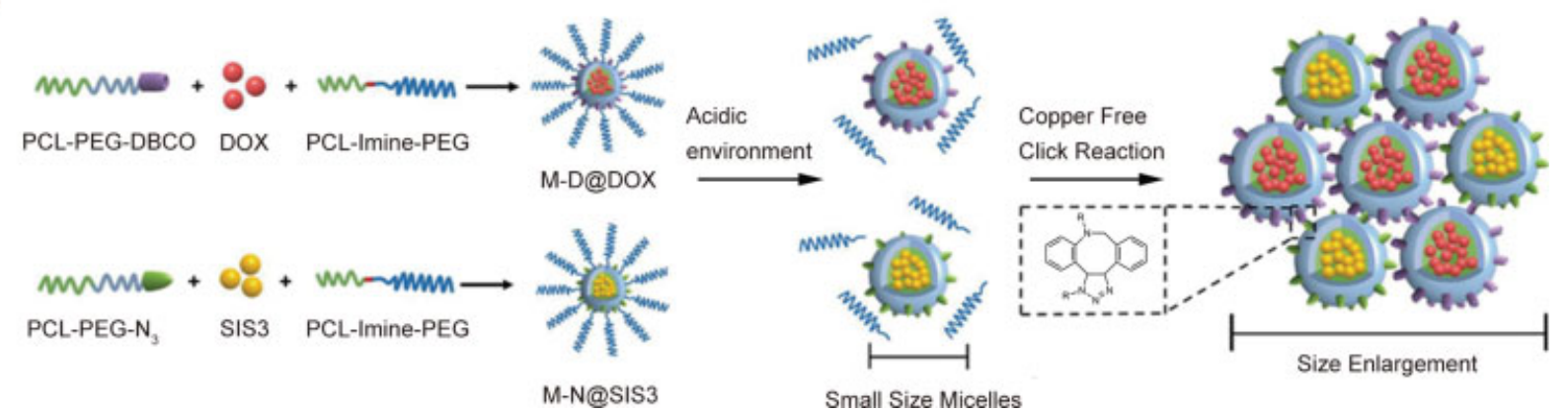

Fig. 5 (A) Design of enhanced accumulation of micelles for cancer immunochemotherapy. M-Dox/MPLA (+), composed of DSPE-PEG-N3 and DSPE-PEG-Alk, is engineered for $\mathrm{Cu}(\mathrm{l})$-catalyzed azide/alkyne cycloaddition with size increase to improve tumor accumulation of drugs. Copyright 2018 American Chemical Society. (B) M-DN@DOX/SIS3 was a mixture of M-D@DOX and M-N@SIS3, which could remain relatively independent in the systemic circulation; once in an acidic environment, the protective PEG5000 coating shed off, leading to the rapid copperfree click reaction to form stable micellar clusters. Copyright 2021 American Chemical Society.

Table 2 Size shrinkage strategies and typical NPs

\begin{tabular}{|c|c|c|c|c|}
\hline Stimuli & Responsive materials & Typical NPs & Size change & Ref. \\
\hline \multirow[t]{2}{*}{$\mathrm{pH}$} & Acid-labile chemical bonds & iCluster/Pt & $\begin{array}{l}\sim 100 \mathrm{~nm} \rightarrow \\
\sim 5 \mathrm{~nm}\end{array}$ & 66 \\
\hline & $\begin{array}{l}\text { Polymers containing ionizable } \\
\text { groups (sulfonamide- and } L- \\
\text { histidine-based polymers, etc.) }\end{array}$ & PAH/RGX-104@PDM/PTX & $\begin{array}{l}\sim 132 \mathrm{~nm} \rightarrow \\
\sim 35 \mathrm{~nm}\end{array}$ & 70,71 \\
\hline \multirow[t]{3}{*}{ Enzyme } & Gelatin (substrates of MMP2) & QDGelNPs & $\begin{array}{l}\sim 100 \mathrm{~nm} \rightarrow \\
\sim 10 \mathrm{~nm}\end{array}$ & 73 \\
\hline & $\begin{array}{l}\text { EGPLGVRGK (substrate peptide } \\
\text { of MMP2) }\end{array}$ & DGL/GEM@PP/GA & $\begin{array}{l}\sim 151 \mathrm{~nm} \rightarrow \\
\sim 28 \mathrm{~nm}, \text { or } 88 \mathrm{~nm}\end{array}$ & 75 \\
\hline & Hyaluronic acid & DA-tMN & $\begin{array}{l}\sim 100 \mathrm{~nm} \rightarrow \\
\sim 10 \mathrm{~nm}\end{array}$ & 78 \\
\hline Redox & Disulfide bond & PSPD/Dex-P123 & $\begin{array}{l}\sim 120 \mathrm{~nm} \rightarrow \\
\sim 30 \mathrm{~nm}\end{array}$ & 81 \\
\hline ROS & $\begin{array}{l}\text { Thioether, thioketal, boronic } \\
\text { ester, selenium, sulfide and } \\
\text { ferrocenyl }\end{array}$ & TK-PPE@NPCe6/DOX & $\begin{array}{l}\sim 154 \mathrm{~nm} \rightarrow \\
\sim 72 \mathrm{~nm}\end{array}$ & $84-87,90$ \\
\hline
\end{tabular}

Abbreviation: NPs, nanoparticles. 
Table 3 Chemical structures and degradation products of acid-labile chemical bonds

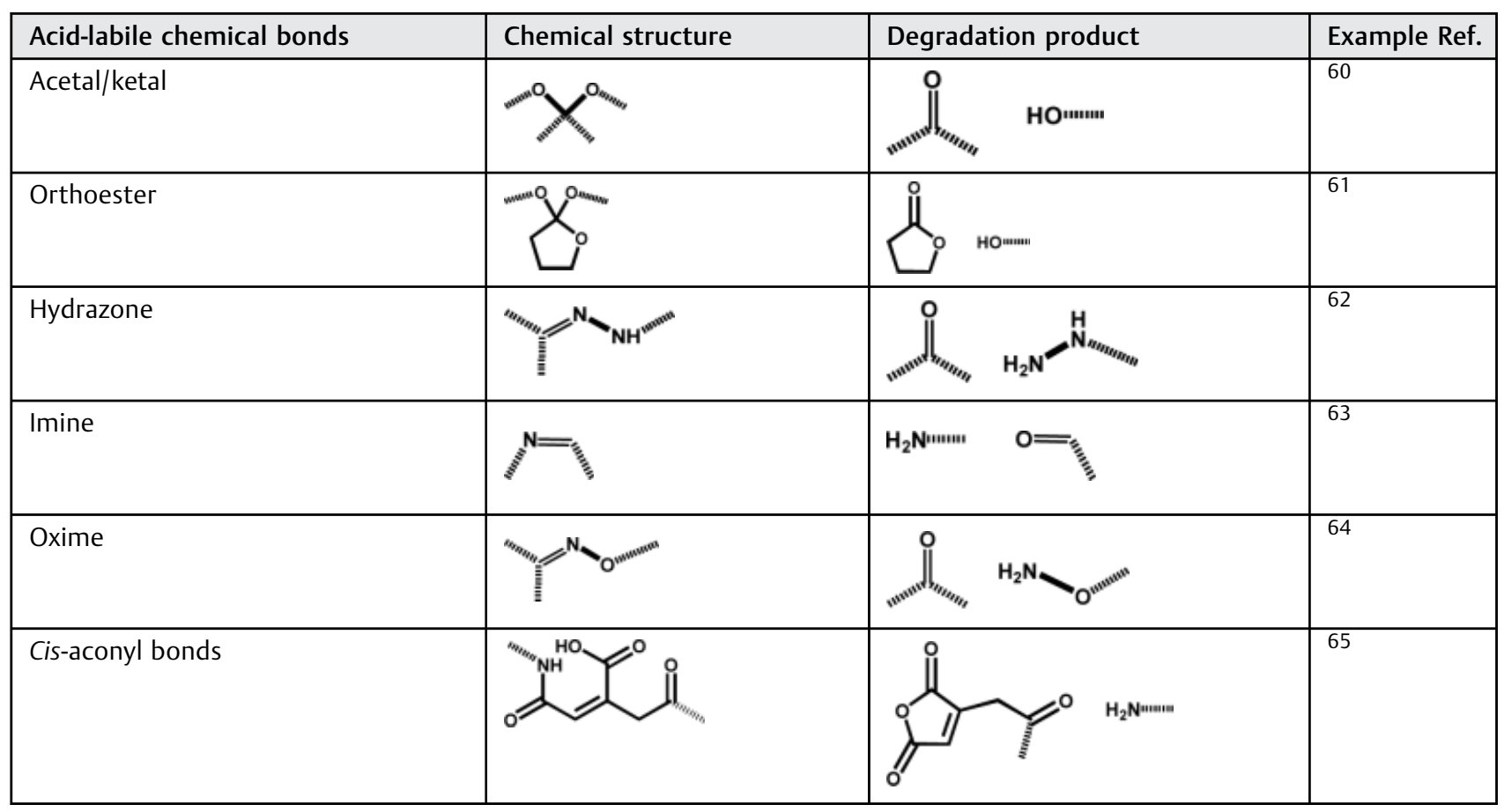

labile amide bond will be cleaved and trigger the release of small PAMAM prodrugs $(\sim 5 \mathrm{~nm})$ for deep penetration ( - Fig. 6A). Other acid-labile chemical bonds are also frequently reported to achieve site-specific drug release or exposure of functional peptides, ${ }^{67,68}$ while their applications in NP size shrinkage are worthy of further development.

Polymers containing ionizable groups could undergo hydrophilic-hydrophobic transitions at different $\mathrm{pH}$ values, the polymer is hydrophilic when ionized, but is hydrophobic when deionized. ${ }^{69}$ In tumor-targeted nano-systems, ionizable groups often act as the hydrophobic segment of block copolymers, which can ionize and becomes hydrophilic in response to an acidic environment, resulting in size shrinkage or disintegration of nano-systems. The most commonly used materials of $\mathrm{pH}-$ sensitive hydrophilic-hydrophobic transformability include sulfonamide- and L-histidine-based polymers. ${ }^{70}$ Our group designed a dual-pH-sensitive size-shrinkable conjugated micelle system (PAH/RGX-104@PDM/PTX) that could simultaneously target tumor perivascular regions and deep tumor areas. ${ }^{71}$ PAH/RGX-104@PDM/PTX consists of two mixed micelles PAH/RGX-104 (containing PEG-PAEMA, DSPE-PEG-SH, and RGX-104) and PDM/PTX (containing PEG-PDPA, DSPE-PEGMal, and paclitaxel [PTX]), which are further conjugated through the Michael addition reaction to form a stable NP of around $132 \mathrm{~nm}$. Once it reaches the acidic tumor microenvironment $(\mathrm{pH}$ 6.8), PAEMA is ionized and becomes hydrophilic, PAH/RGX-104 disintegrates to release RGX-104 in the perivascular area. At the same time, PDM/PTX keeps intact, NP size shrinks to around $35 \mathrm{~nm}$ and is allowed for deep tumor penetration. After uptaken by tumor cells, PDPA undergoes ionization in responsive response to the endo/lysosome (pH 5.6) and becomes hydrophilic, PDM/PTX disintegrates to release PTX ( - Fig. 6B).

\section{Enzyme-Triggered Size Shrinkage}

Tumor-associated enzyme-triggered size shrinkage is one of the most commonly used and effective strategies to achieve efficient tumor targeting and tumor penetration. There are a variety of overexpressed enzymes in the tumor microenvironment, such as MMPs and HAase. MMPs such as MMP-2 are generally secreted by tumor cells for degrading the ECM and support cancer angiogenesis, progression, metastasis, and invasion. ${ }^{22,72}$ Gelatin and collagens are the main substrates that can be recognized and degraded by MMP-2, thus are frequently introduced in MMP-2-responsive size-tunable drug delivery nano-systems. Wong et al constructed a multistage QD gelatin NP (QDGelNP) with initial size of around $100 \mathrm{~nm} .{ }^{73}$ After exuding from tumor blood vessels into the tumor microenvironment, the core gelatin NPs of QDGelNPs were degraded by the highly expressed MMP-2 in the tumor microenvironment, releasing smaller $10-\mathrm{nm}$ particle QDs (-Fig. 7A). This multistage size-shrinkable NP not only has long blood circulation to facilitate tumor accumulation based on the EPR effect, but also can overcome the dense collagen matrix barrier to achieve deep tumor penetration. In addition to deep tumor penetration, an important mission of the size-shrinkage strategy is to achieve hierarchical targeting at tumor sites. In tumor microenvironment, tumor-associated fibroblasts (TAFs) form a predominant stromal cellular component and hinder the delivery of nano-drugs to deep tumor cells. $^{74}$ Our group proposed a multifunctional size-shrinkable nanoplatform DGL/GEM@PP/GA for TAF-targeted regulation and deep tumor penetration. ${ }^{75}$ Gemcitabine (GEM)-conjugated dendrigraft poly-L-lysine (DGL, a dendrimer of small particle size) was connected to poly(ethylene glycol)-poly(caprolactone) (PEG-PCL) through the substrate 
A

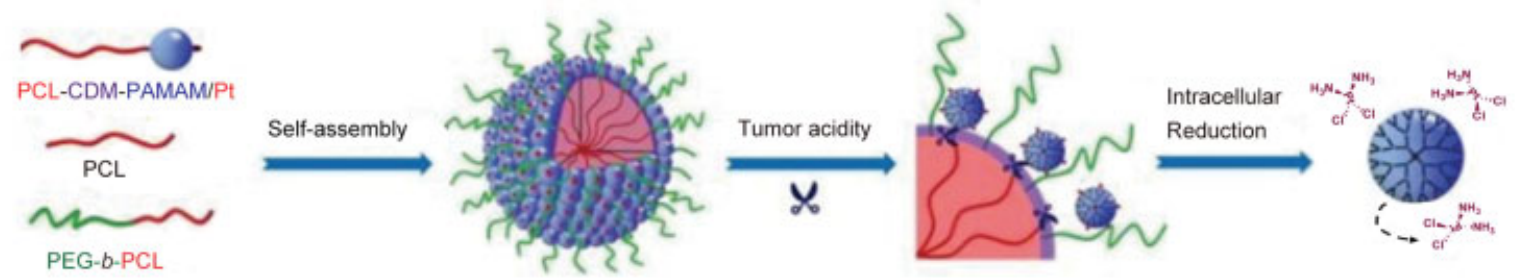

B

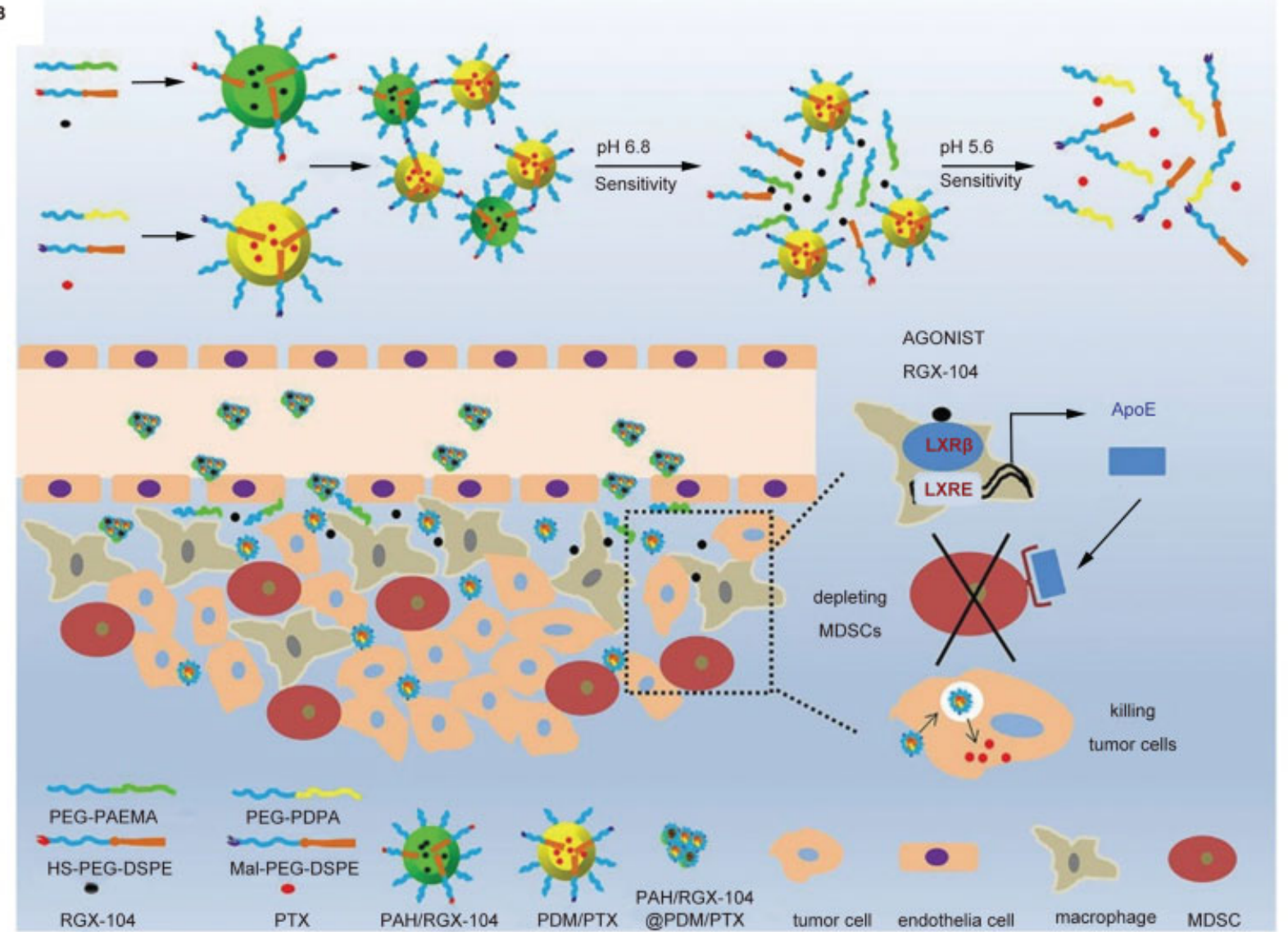

Fig. 6 (A) Self-assembly and structural change of iCluster/Pt in response to tumor acidity and intracellular reductive environment. Copyright 2016 National Academy of Sciences. (B) Structures of the conjugated micelle system and schematic illustration of the construction and the transformation of PAH/RGX-104@PDM/PTX. The enhanced intratumoral accumulation and penetration resulted from the transformation and the simultaneous combinational therapy are also shown in the scheme. Copyright 2019 Elsevier B.V. All rights reserved.

peptide of MMP-2 (EGPLGVRGK). DGL/GEM@PP/GA $(\sim 151 \mathrm{~nm})$ could release small DGL/GEM $(<50 \mathrm{~nm})$ in response to MMP-2 for deep tumor penetration, leaving $18 \beta$ glycyrrhetinic acid (GA)-loaded large NPs $(>50 \mathrm{~nm})$ around tumor vessels to regulate the TAFs ( - Fig. 7B). By adopting the hierarchical targeting strategy of tumor cells and TAFs, DGL/GEM@PP/GA showed significant antitumor effects against pancreatic cancer and breast cancer. To further improve the accuracy of hierarchical targeting and the therapeutic efficacy of tumors, our group designed a novel size-shrinkable NP HSA-PTX@CAP-ITSL, which was responsive to the membrane biomarker fibroblast activation protein- $\alpha$ (FAP- $\alpha)$ on cancer-associated fibroblasts and NIR laser irradiation. ${ }^{76}$ A small-sized albumin NP of PTX (HSA-PTX) was encapsulated into the CAP-(a substrate peptide of FAP- $\alpha$ ) modified IR-780-incorporated thermosensitive liposomes
(CAP-ITSL) to form the HSA-PTX@CAP-ITSL with an initial size of $123.9 \pm 1.9 \mathrm{~nm}$. Upon arrival at the tumor microenvironment, FAP- $\alpha$ triggered the release of HSA-PTX with a small size of around $30 \mathrm{~nm}$ for deep tumor penetration; meanwhile, a sequential stimulation of NIR laser irradiation produced hyperthermia to kill tumor cells and also expand the tumor interstitial space, which further promoted the release and penetration of HSA-PTX (- Fig. 7C).

Similar to MMPs, HAase is an abundant component of tumor microenvironment, which can specifically hydrolyze HA. HA also possesses excellent CD44 (a receptor overexpressed on certain tumor cell surface) targeting ability. ${ }^{77} \mathrm{By}$ using the HA nanogel as the core NP and modifying the doxorubicin (DOX) and AP-18 (a transient receptor potential ankyrin 1 inhibitor) co-loaded tLyP-1-modified DSPE-PEG 2000 micelles on its surface, our group developed a dual receptor- 
A

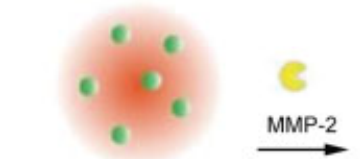
preferentially accumulate in solid tumors by EPR

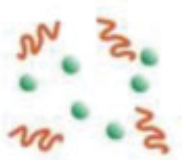

$10 \mathrm{~nm}$ QDs

can penetrate deep into

tumor tissue

B

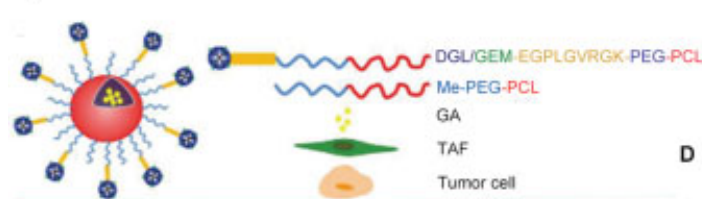

D
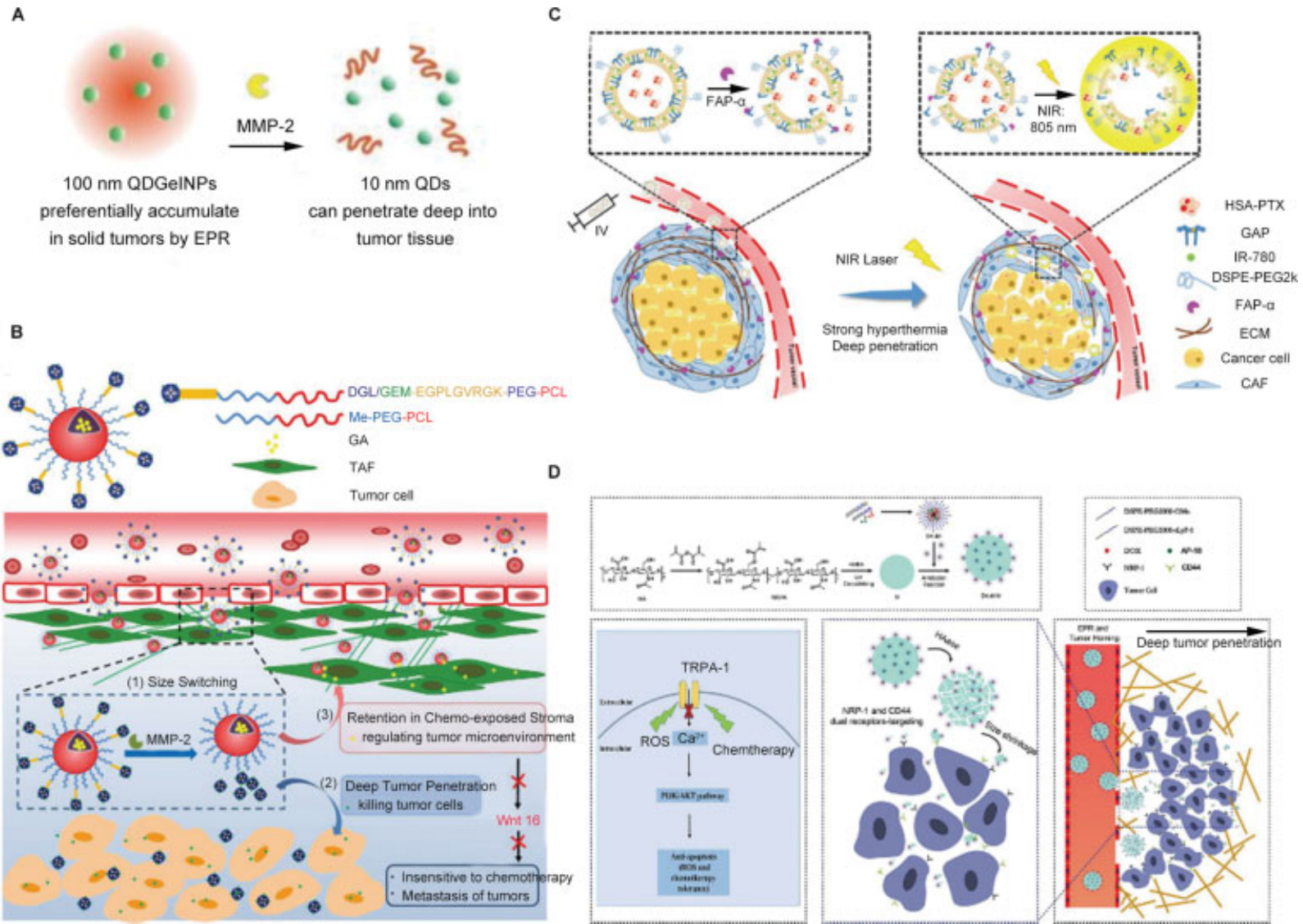

?
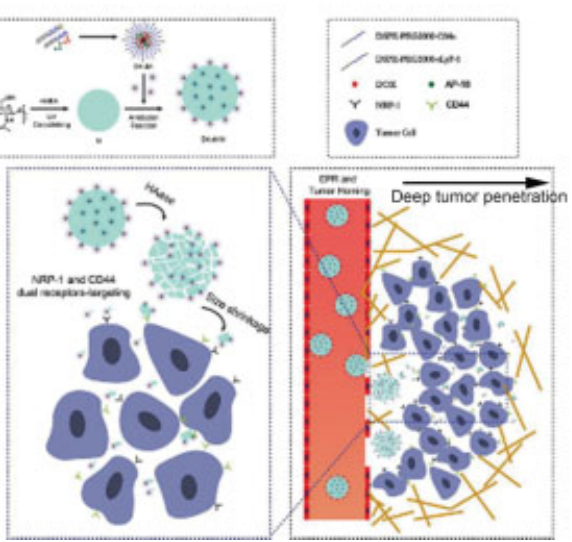

Fig. 7 (A) Schematic of 100-nm QDGelNPs changing size to 10-nm QD NPs by cleaving away the gelatin scaffold with MMP-2, a protease highly expressed in tumor tissue. Copyright 2011 National Academy of Sciences. (B) Schematic Illustration of DGL/GEM@PP/GA for deep tumor penetration and tumor microenvironment regulation. Copyright 2019 American Chemical Society. (C) Diagram of dual-responsive lipid-albumin NPs (HSA-PTX@CAP-ITSL) for deep tumor penetration and combined therapy against pancreatic tumors. Copyright 2020 Elsevier B.V. All rights reserved. (D) Schematic illustration of DA-tMN delivered into tumor. Copyright 2020 Elsevier B.V. All rights reserved. NPs, nanoparticles; MMP-2, matrix metalloproteinase-2.

targeting and size-switchable "cluster bomb" DA-tMN. ${ }^{78}$ The degradation of core HA nanogels in responsive to HAase could realize the release of small-sized DSPE-PEG 2000 micelles and the transition of size from $\sim 100$ to $\sim 10 \mathrm{~nm}$ ( - Fig. 7D). Although the enzyme-triggered size-switchable strategy has become a research hotspot, the heterogeneity of the expression levels of enzymes in different tumors, which limits the application scope of NPs, is still worthy of attention.

\section{Redox-Induced Size Shrinkage}

The concentration of intracellular glutathione (GSH) in the tumor microenvironment is significantly higher than that in the extracellular circulation and fluids, ${ }^{79}$ thus is regarded as a promising point to achieve reduction-sensitive drug delivery of tumor. Disulfide bond is a kind of chemical linkage that is easy to degrade in the reduction potential environment. ${ }^{80}$ Wang et al utilized disulfide bond to conjugate the amphiphilic blocks (termed P123) to charge-reversible blocks (termed DMMA-PEI) to form a pH- and redox-sensitive size-tunable NP PSPD. ${ }^{81}$ And further incorporated a dexamethasone (Dex)conjugated P123 (denoted as P123-Dex) and encapsulated DOX through hydrophobic interactions. In GSH-elevated intracellular cytosol of cancer cells, the disulfide bond was cleaved to detach polyethylenimine (PEI) from the micelle surface, triggering the size shrinkage of the hybrid micelles from $\sim 120$ to $\sim 30 \mathrm{~nm}$. And the small-sized NPs were allowed to deliver DOX into cell nuclei through nuclear pores ( - Fig. 8A). Guo et al designed another size-shrinkable micellar nano-system based on mPEG-PLA-ss-PEI-DMMA (PELEss-DA) polymer, which facilitates direct drug delivery into the nucleus for therapy of MDR tumor cells. ${ }^{82}$ The PELEss-DA first increases in the size under acidic $\mathrm{pH}$ conditions of the tumor microenvironment via the charge reversal of DMMA, then is internalized by the tumor cells and altered to smaller micelles triggered by intracellular GSH ( $\mathbf{- F i g . ~ 8 B )}$.

\section{ROS-Triggered Size Shrinkage}

Oxidization-based size change is usually triggered by the high content of ROS in tumor cells. ROS, including hydrogen peroxides $\left(\mathrm{H}_{2} \mathrm{O}_{2}\right)$, superoxides $\left(\mathrm{O}_{2}^{-}\right)$, singlet oxygen $\left({ }^{1} \mathrm{O}_{2}\right)$, and hydroxyl radicals $(\mathrm{OH} \cdot)$, are $\sim 100$ times higher in tumor cells than that in normal cells due to a series of oncogenic transformations. ${ }^{83}$ ROS-responsive groups mainly contain thioether, thioketal, boronic ester, selenium, sulfide, and ferrocenyl. ${ }^{84-87}$ However, it is difficult to achieve efficient oxidation-derived chemical bond hydrolysis only relying on the original amount of ROS in tumor cells. Photodynamic therapy (PDT), which is facilitated by generating ROS to 
A

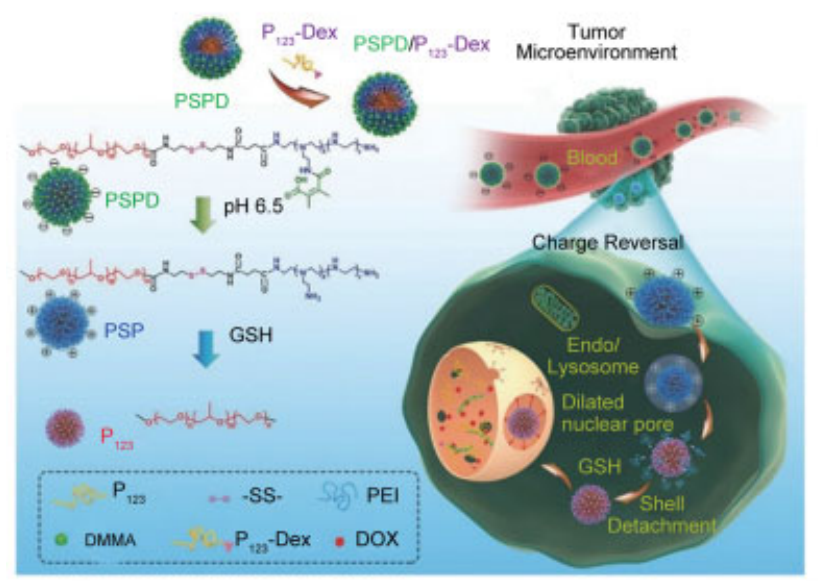

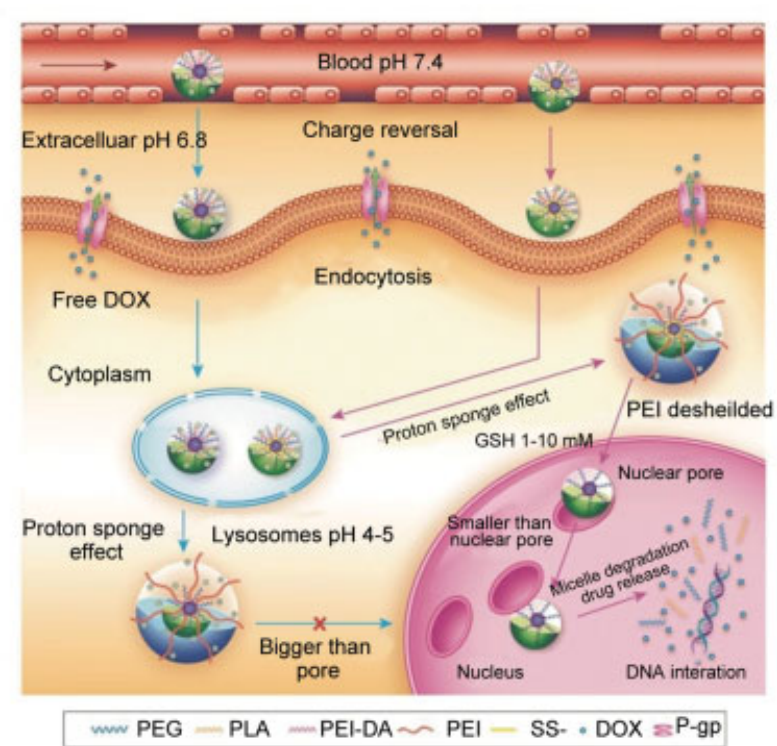

Fig. 8 (A) Schematic illustration of the cooperative dimensional strategy for anticancer drug delivery mediated by hybrid micelle PSPD/P123-Dex. By exploiting tumoral $\mathrm{pH}$ and intracellular redox potential, as well as the ability of Dex to target and dilate nuclear pores, PSPD/P123-Dex can efficiently deliver DOX into the nucleus of tumor cells. Copyright 2017 WILEY-VCH Verlag GmbH \& Co. KGaA, Weinheim. (B) Schematic design of the nucleus entry of size-changeable polymer micelles (PELEss-DA) to overcome MDR. Copyright 2015 WILEY-VCH Verlag $\mathrm{GmbH} \& \mathrm{Co}$., KGaA, Weinheim. Dex, dexamethasone; MDR, multidrug resistant.

induce cell apoptosis by photosensitizers under red or NIR light irradiation, ${ }^{88,89}$ has great potential to be combined with ROS-responsive materials for enhanced tumor drug delivery. Cao et al constructed a ROS-sensitive polymeric nanocarrier TK-PPE@NP ${ }_{C e 6 / D o x}$ to achieve remotely controlled drug release by light-activated size shrinkage. ${ }^{90}$ The TK-PPE@NP ${ }_{\mathrm{Ce} /}$ Dox consists of four parts: ROS-responsive poly(thioketal phosphoester) (TK-PPE), amphiphilic diblock copolymer PEG-b-PCL, photosensitizer Ce6, and chemotherapeutic drug DOX. Under 660-nm red light irradiation, the TK-PPE core was rapidly degraded into oligomers or small molecules by Ce6-derived ROS, consequently, the NP size shrank from $154 \pm 4$ to $72 \pm 3 \mathrm{~nm}$, which triggered the release of encapsulated DOX (-Fig. 9).

\section{Conclusion and Perspectives}

With the development of cognition in the field of oncology, tremendous progress has been achieved in the design of nano-drug delivery systems in recent years. The awareness of tumor retention and penetration has led to the emergence of size-adjustable nano-drug delivery systems. And by using the external stimuli or the unique characteristics of the tumor microenvironment and tumor cells, a variety of stimuli-responsive size-adjustable nano-drug delivery systems have been reported. This review summarizes the principles and applications of size-adjustable nano-drug delivery systems triggered by acidic $\mathrm{pH}$, overexpressed enzymes, temperature, light, catalysts, redox environment, and ROS. However, there are still many challenges in scientific research and in clinical application.

On the one hand, tumors possess complex heterogeneity. The internal physiological properties of tumors of different types, different stages of progression, and different pathological environments are very different, hence the sensitivity of NP transformation will be greatly affected. On the other hand, some tumor-characteristic endogenous stimuli, such as receptors, enzymes, and acidic $\mathrm{pH}$, are not only exist in tumors, but more or less exist in normal tissues, which will cause the off-target of drugs. In addition, the application of exogenous stimuli is limited by the type of tumor, just as light stimulation is mostly only suitable for superficial tumors. Facing the complex tumor microenvironment, the design of the drug delivery systems should be performed after fully understanding the biological characteristics of tumors, the application range and time of the drug delivery systems should be carefully selected, and more ligand modification can be used to enhance the active targeting of NPs to tumor sites.

In terms of clinical application, the increasing controversy of EPR effect makes the possibility of clinical transformation of EPR-effect-based NPs greatly reduced. The EPR effect shows good effect in animal models but the performance in clinical trials is not satisfactory, which mainly attribute to the heterogeneity of the EPR effect. ${ }^{91}$ The EPR effect is highly dependent on tumor permeability and perfusion and IFP. In murine models, tumors grow rapidly, their vasculature is abnormally developed and have higher permeability, perfusion, and lower IFP. While in humans, lack of fenestrations in the tumor endothelium, lower pericyte coverage, higher density of the ECM, etc., lead to higher IFP and lower permeability and perfusion. ${ }^{92}$ Facing the heterogeneity of the EPR effect, the use of active targeting strategies or the remodeling of the tumor microenvironment by PDT, sonodynamic therapy, radiation therapy, etc., to improve the EPR effect are promising solutions. 
A

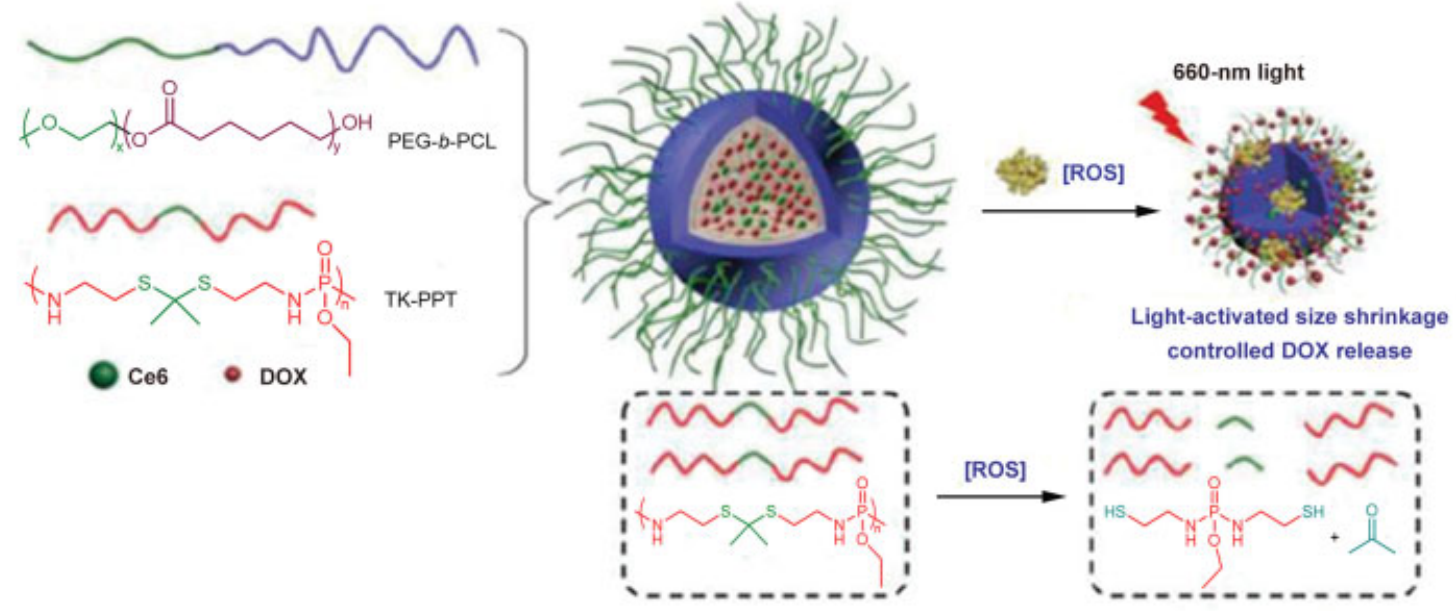

B

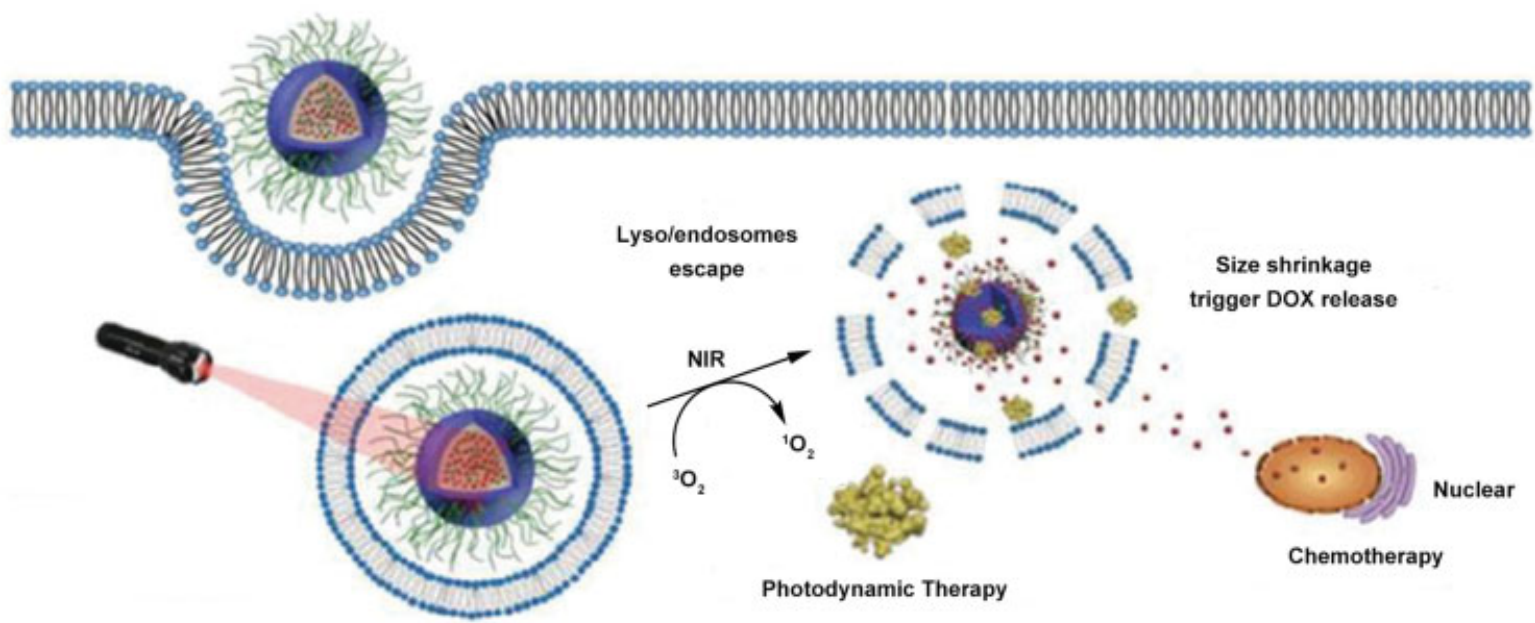

Fig 9 (A) Formation and mechanism of light-activated shrinkable nanoparticle TK-PPE@NPCe6/DOX. (B) Schematic illustration of TKPPE@NPCe6/DOX with light-activated size shrinkage for controlled DOX release. Copyright 2017 American Chemical Society. DOX, doxorubicin.

In general, to further improve the therapeutic effect and the possibility of clinical transformation of the size-adjustable nano-drug delivery systems, the biological characteristics of tumors should be fully explored, and the design of NPs should be precise, personalized, and simplified. And we believe, there is a broad prospect with efforts of numerous researchers.

Funding

We are very grateful for the support from the National Natural Science Foundation (Grant No. 81773658), the Program Sichuan Veterinary Medicine and Drug Innovation Group of China Agricultural Research System (Grant No. SCCXTD-2020-18), and Sichuan University Postdoctoral Interdisciplinary Innovation Fund.

Conflict of Interest

The authors declare no conflict of interest.

\section{References}

1 Fang J, Nakamura H, Maeda H. The EPR effect: unique features of tumor blood vessels for drug delivery, factors involved, and limitations and augmentation of the effect. Adv Drug Deliv Rev 2011;63(03):136-151

2 Li C, Wang J, Wang Y, et al. Recent progress in drug delivery. Acta Pharm Sin B 2019;9(06):1145-1162

3 Yang Z, Chen Q Chen J, et al. Tumor-pH-responsive dissociable albumin-tamoxifen nanocomplexes enabling efficient tumor penetration and hypoxia relief for enhanced cancer photodynamic therapy. Small 2018;14(49):e1803262

4 Duan X, Li Y. Physicochemical characteristics of nanoparticles affect circulation, biodistribution, cellular internalization, and trafficking. Small 2013;9(9-10):1521-1532

5 Perrault SD, Walkey C, Jennings T, Fischer HC, Chan WC. Mediating tumor targeting efficiency of nanoparticles through design. Nano Lett 2009;9(05):1909-1915

6 Mei L, Liu Y, Rao J, et al. Enhanced tumor retention effect by click chemistry for improved cancer immunochemotherapy. ACS Appl Mater Interfaces 2018;10(21):17582-17593

7 Ruan S, Hu C, Tang X, et al. Increased gold nanoparticle retention in brain tumors by in situ enzyme-induced aggregation. ACS Nano 2016;10(11):10086-10098

8 Larsen EK, Nielsen T, Wittenborn T, et al. Size-dependent accumulation of PEGylated silane-coated magnetic iron oxide nanoparticles in murine tumors. ACS Nano 2009;3(07):1947-1951

9 Du J, Xu N, Fan J, Sun W, Peng X. Carbon dots for in vivo bioimaging and theranostics. Small 2019;15(32):e1805087 
10 Farzin A, Etesami SA, Quint J, Memic A, Tamayol A. Magnetic nanoparticles in cancer therapy and diagnosis. Adv Healthc Mater 2020;9(09):e1901058

11 Chaturvedi VK, Singh A, Singh VK, Singh MP. Cancer nanotechnology: a new revolution for cancer diagnosis and therapy. Curr Drug Metab 2019;20(06):416-429

12 Jain RK, Stylianopoulos T. Delivering nanomedicine to solid tumors. Nat Rev Clin Oncol 2010;7(11):653-664

13 Gupta P, Vermani K, Garg S. Hydrogels: from controlled release to pH-responsive drug delivery. Drug Discov Today 2002;7(10): 569-579

14 Liu X, Chen Y, Li H, et al. Enhanced retention and cellular uptake of nanoparticles in tumors by controlling their aggregation behavior. ACS Nano 2013;7(07):6244-6257

$15 \mathrm{Wu} \mathrm{W}$, Li S, Lin Z, Li J. pH-sensitive nanocarriers for enhanced tumor retention and rapid intracellular drug release. J Control Release 2015;213:e111-e112

16 Krebs MR, Domike KR, Donald AM. Protein aggregation: more than just fibrils. Biochem Soc Trans 2009;37(Pt 4):682-686

17 Li H, Chen Y, Li Z, Li X, Jin Q, Ji J. Hemoglobin as a smart pHsensitive nanocarrier to achieve aggregation enhanced tumor retention. Biomacromolecules 2018;19(06):2007-2013

18 Ganta S, Devalapally H, Shahiwala A, Amiji M. A review of stimuliresponsive nanocarriers for drug and gene delivery. J Control Release 2008;126(03):187-204

19 Qiu Y, Park K. Environment-sensitive hydrogels for drug delivery. Adv Drug Deliv Rev 2001;53(03):321-339

20 Zhang W, Lin Y. The mechanism of asparagine endopeptidase in the progression of malignant tumors: a review. Cells 2021;10(05):1153

21 Spinelli FM, Vitale DL, Sevic I, Alaniz L. Hyaluronan in the tumor microenvironment. Adv Exp Med Biol 2020;1245:67-83

22 Kessenbrock K, Plaks V, Werb Z. Matrix metalloproteinases: regulators of the tumor microenvironment. Cell 2010;141(01):52-67

23 Mijanović O, Branković A, Panin AN, et al. Cathepsin B: a sellsword of cancer progression. Cancer Lett 2019;449:207-214

24 Jaaks P, Bernasconi M. The proprotein convertase furin in tumour progression. Int J Cancer 2017;141(04):654-663

25 Peng Z, Chang Y, Fan J, Ji W, Su C. Phospholipase A2 superfamily in cancer. Cancer Lett 2021;497:165-177

26 Chen JM, Dando PM, Rawlings ND, et al. Cloning, isolation, and characterization of mammalian legumain, an asparaginyl endopeptidase. J Biol Chem 1997;272(12):8090-8098

27 Liu C, Sun C, Huang H, Janda K, Edgington T. Overexpression of legumain in tumors is significant for invasion/metastasis and a candidate enzymatic target for prodrug therapy. Cancer Res 2003; 63(11):2957-2964

28 Liu Z, Xiong M, Gong J, et al. Legumain protease-activated TATliposome cargo for targeting tumours and their microenvironment. Nat Commun 2014;5:4280

29 Zhou H, Sun H, Lv S, et al. Legumain-cleavable 4-arm poly(ethylene glycol)-doxorubicin conjugate for tumor specific delivery and release. Acta Biomater 2017;54:227-238

$30 \mathrm{He}$ X, Cao H, Wang $\mathrm{H}$, et al. Inflammatory monocytes loading protease-sensitive nanoparticles enable lung metastasis targeting and intelligent drug release for anti-metastasis therapy. Nano Lett 2017;17(09):5546-5554

31 Toole BP. Hyaluronan and its binding proteins, the hyaladherins. Curr Opin Cell Biol 1990;2(05):839-844

32 Stern R. Hyaluronan catabolism: a new metabolic pathway. Eur J Cell Biol 2004;83(07):317-325

33 Stern R. Hyaluronidases in cancer biology. Semin Cancer Biol 2008;18(04):275-280

$34 \mathrm{Hu} \mathrm{Q}$ Sun W, Lu Y, et al. Tumor microenvironment-mediated construction and deconstruction of extracellular drug-delivery depots. Nano Lett 2016;16(02):1118-1126

35 Jochum FD, Theato P. Temperature- and light-responsive smart polymer materials. Chem Soc Rev 2013;42(17):7468-7483
36 Schild HG. Poly(N-isopropylacrylamide): experiment. Theory and Application Prog. Polym Sci 1992;17(02):163-249

37 Idziak I, Avoce D, Lessard D, Gravel D, Zhu XX. Thermosensitivity of aqueous solutions of poly(N,N-diethylacrylamide). Macromolecules 1999;32:1260-1263

38 Lutz JF, Hoth A. Preparation of ideal PEG analogues with a tunable thermosensitivity by controlled radical copolymerization of 2-(2methoxyethoxy)ethyl methacrylate and oligo(ethylene glycol) methacrylate. Macromolecules 2006;39:893-896

39 Qiao SL, Ma Y, Wang Y, et al. General approach of stimuli-induced aggregation for monitoring tumor therapy. ACS Nano 2017;11 (07):7301-7311

40 Liu FH, Cong Y, Qi GB, Ji L, Qiao ZY, Wang H. Near-infrared laserdriven in situ self-assembly as a general strategy for deep tumor therapy. Nano Lett 2018;18(10):6577-6584

41 Melancon MP, Zhou M, Li C. Cancer theranostics with nearinfrared light-activatable multimodal nanoparticles. Acc Chem Res 2011;44(10):947-956

42 Hajebi S, Rabiee N, Bagherzadeh M, et al. Stimulus-responsive polymeric nanogels as smart drug delivery systems. Acta Biomater 2019;92:1-18

43 Liu G, Liu W, Dong CM. UV- and NIR-responsive polymeric nanomedicines for on-demand drug delivery. Polym Chem-UK 2013;4(12):3431-3443

$44 \mathrm{Lu}$ Y, Lin Y, Chen Z, et al. Enhanced endosomal escape by lightfueled liquid-metal transformer. Nano Lett 2017;17(04): 2138-2145

45 Shiraishi Y, Shirakawa E, Tanaka K, Sakamoto H, Ichikawa S, Hirai T. Spiropyran-modified gold nanoparticles: reversible size control of aggregates by UV and visible light irradiations. ACS Appl Mater Interfaces 2014;6(10):7554-7562

46 Manna A, Chen PL, Akiyama H, Wei TX, Tamada K, Knoll W. Optimized photoisomerization on gold nanoparticles capped by unsymmetrical azobenzene disulfides. Chem Mater 2003;15(01): 20-28

47 Cheng X, Sun R, Yin L, Chai Z, Shi H, Gao M. Light-triggered assembly of gold nanoparticles for photothermal therapy and photoacoustic imaging of tumors in vivo. Adv Mater 2017;29(06): 201604894

48 Kuk S, Lee BI, Lee JS, Park CB. Rattle-structured upconversion nanoparticles for near-IR-induced suppression of Alzheimer's $\beta$ amyloid aggregation. Small 2017;13(11):1603139

49 Nyk M, Kumar R, Ohulchanskyy TY, Bergey EJ, Prasad PN. High contrast in vitro and in vivo photoluminescence bioimaging using near infrared to near infrared up-conversion in Tm3 + and $\mathrm{Yb} 3+$ doped fluoride nanophosphors. Nano Lett 2008;8(11): 3834-3838

50 Zhao T, Wang P, Li Q et al. Near-infrared triggered decomposition of nanocapsules with high tumor accumulation and stimuli responsive fast elimination. Angew Chem Int Ed Engl 2018;57 (10):2611-2615

51 Alonso F, Moglie Y, Radivoy G. Copper nanoparticles in click chemistry. Acc Chem Res 2015;48(09):2516-2528

52 Liang L, Astruc D. The copper(I)-catalyzed alkyne-azide cycloaddition (CuAAC) "click" reaction and its applications. An overview. Coord Chem Rev 2011;255(23-24):2933-2945

53 Ning X, Guo J, Wolfert MA, Boons GJ. Visualizing metabolically labeled glycoconjugates of living cells by copper-free and fast huisgen cycloadditions. Angew Chem Int Ed Engl 2008;47(12): 2253-2255

54 Dommerholt J, Schmidt S, Temming R, et al. Readily accessible bicyclononynes for bioorthogonal labeling and three-dimensional imaging of living cells. Angew Chem Int Ed Engl 2010;49(49): 9422-9425

55 Lee $\mathrm{S}$, Koo $\mathrm{H}$, Na JH, et al. Chemical tumor-targeting of nanoparticles based on metabolic glycoengineering and click chemistry. ACS Nano 2014;8(03):2048-2063 
56 Rondon A, Degoul F. Antibody pretargeting based on bioorthogonal click chemistry for cancer imaging and targeted radionuclide therapy. Bioconjug Chem 2020;31(02):159-173

57 Deng M, Guo R, Zang S, et al. pH-Triggered copper-free click reaction-mediated micelle aggregation for enhanced tumor retention and elevated immuno-chemotherapy against melanoma. ACS Appl Mater Interfaces 2021;13(15):18033-18046

58 Panté N, Kann M. Nuclear pore complex is able to transport macromolecules with diameters of about $39 \mathrm{~nm}$. Mol Biol Cell 2002;13(02):425-434

59 Chen B, Dai W, He B, et al. Current multistage drug delivery systems based on the tumor microenvironment. Theranostics 2017;7(03):538-558

60 Liu J, Huang Y, Kumar A, et al. pH-sensitive nano-systems for drug delivery in cancer therapy. Biotechnol Adv 2014;32(04): 693-710

61 Thambi T, Deepagan VG, Chang KY, Chang KY, Park JH. Synthesis and physicochemical characterization of amphiphilic block copolymers bearing acid-sensitive orthoester linkage as the drug carrier. Polymer (Guildf) 2011;52(21):4753-4759

62 Ding M, Song N, He X, et al. Toward the next-generation nanomedicines: design of multifunctional multiblock polyurethanes for effective cancer treatment. ACS Nano 2013;7(03):1918-1928

63 Gurski LA, Jha AK, Zhang C, Jia X, Farach-Carson MC. Hyaluronic acid-based hydrogels as 3D matrices for in vitro evaluation of chemotherapeutic drugs using poorly adherent prostate cancer cells. Biomaterials 2009;30(30):6076-6085

64 Jin Y, Song L, Su Y, et al. Oxime linkage: a robust tool for the design of pH-sensitive polymeric drug carriers. Biomacromolecules 2011;12(10):3460-3468

65 Hu FQ, Zhang YY, You J, Yuan H, Du YZ. pH triggered doxorubicin delivery of PEGylated glycolipid conjugate micelles for tumor targeting therapy. Mol Pharm 2012;9(09):2469-2478

66 Li HJ, Du JZ, Du XJ, et al. Stimuli-responsive clustered nanoparticles for improved tumor penetration and therapeutic efficacy. Proc Natl Acad Sci U S A 2016;113(15):4164-4169

67 Li L, Sun W, Zhong J, et al. Multistage nanovehicle delivery system based on stepwise size reduction and charge reversal for programmed nuclear targeting of systemically administered anticancer drugs. Adv Funct Mater 2015;25:4101-4113

68 Koren E, Apte A, Jani A, Torchilin VP. Multifunctional PEGylated 2C5-immunoliposomes containing $\mathrm{pH}$-sensitive bonds and TAT peptide for enhanced tumor cell internalization and cytotoxicity.J Control Release 2012;160(02):264-273

69 Min SK, Su JH, Han JK, et al. pH-Responsive PEG-Poly( $\beta$-amino ester) block copolymer micelles with a sharp transition. Macromol Rapid Commun 2006;27(06):447-451

70 Lee ES, Shin HJ, Na K, Bae YH. Poly(L-histidine)-PEG block copolymer micelles and $\mathrm{pH}$-induced destabilization. J Control Release 2003;90(03):363-374

71 Wan D, Yang Y, Liu Y, et al. Sequential depletion of myeloidderived suppressor cells and tumor cells with a dual-pH-sensitive conjugated micelle system for cancer chemoimmunotherapy. J Control Release 2020;317:43-56

72 Egeblad M, Werb Z. New functions for the matrix metalloproteinases in cancer progression. Nat Rev Cancer 2002;2(03):161-174

73 Wong C, Stylianopoulos T, Cui J, et al. Multistage nanoparticle delivery system for deep penetration into tumor tissue. Proc Natl Acad Sci U S A 2011;108(06):2426-2431
74 Miao L, Lin CM, Huang L. Stromal barriers and strategies for the delivery of nanomedicine to desmoplastic tumors. J Control Release 2015;219:192-204

75 Cun X, Chen J, Li M, et al. Tumor-associated fibroblast-targeted regulation and deep tumor delivery of chemotherapeutic drugs with a multifunctional size-switchable nanoparticle. ACS Appl Mater Interfaces 2019;11(43):39545-39559

76 Yu Q Qiu Y, Li J, et al. Targeting cancer-associated fibroblasts by dualresponsive lipid-albumin nanoparticles to enhance drug perfusion for pancreatic tumor therapy. J Control Release 2020;321:564-575

77 Menzel EJ, Farr C. Hyaluronidase and its substrate hyaluronan: biochemistry, biological activities and therapeutic uses. Cancer Lett 1998;131(01):3-11

78 Wang Y, Yin S, Mei L, et al. A dual receptors-targeting and sizeswitchable "cluster bomb" co-loading chemotherapeutic and transient receptor potential ankyrin 1 (TRPA-1) inhibitor for treatment of triple negative breast cancer. J Control Release 2020;321:71-83

79 Wang L, Huo M, Chen Y, Shi J. Tumor microenvironment-enabled nanotherapy. Adv Healthc Mater 2018;7(08):e1701156

80 Yang D, Chen W, Hu J. Design of controlled drug delivery system based on disulfide cleavage trigger. J Phys Chem B 2014;118(43): 12311-12317

81 Wang $\mathrm{H}$, Li Y, Bai H, et al. A cooperative dimensional strategy for enhanced nucleus-targeted delivery of anticancer drugs. Adv Funct Mater 2017;27:1700339

82 Guo X, Wei X, Jing Y, Zhou S. Size changeable nanocarriers with nuclear targeting for effectively overcoming multidrug resistance in cancer therapy. Adv Mater 2015;27(41):6450-6456

83 Waris G, Ahsan H. Reactive oxygen species: role in the development of cancer and various chronic conditions. J Carcinog 2006;5:14

84 Sun C, Liang Y, Hao N, et al. A ROS-responsive polymeric micelle with a $\pi$-conjugated thioketal moiety for enhanced drug loading and efficient drug delivery. Org Biomol Chem 2017;15(43):9176-9185

85 Luo CQ Zhou YX, Zhou TJ, et al. Reactive oxygen species-responsive nanoprodrug with quinone methides-mediated GSH depletion for improved chlorambucil breast cancers therapy. J Control Release 2018;274:56-68

86 Gupta MK, Meyer TA, Nelson CE, Duvall CL. Poly(PS-b-DMA) micelles for reactive oxygen species triggered drug release. J Control Release 2012;162(03):591-598

87 Staff RH, Gallei M, Mazurowski M, et al. Patchy nanocapsules of poly(vinylferrocene)-based block copolymers for redox-responsive release. ACS Nano 2012;6(10):9042-9049

88 Dolmans DE, Fukumura D, Jain RK. Photodynamic therapy for cancer. Nat Rev Cancer 2003;3(05):380-387

89 He C, Duan X, Guo N, et al. Core-shell nanoscale coordination polymers combine chemotherapy and photodynamic therapy to potentiate checkpoint blockade cancer immunotherapy. Nat Commun 2016;7:12499

90 Cao Z, Ma Y, Sun C, et al. ROS-sensitive polymeric nanocarriers with red light-activated size shrinkage for remotely controlled drug release. Chem Mater 2018;30(02):517-525

91 Park J, Choi Y, Chang H, Um W, Ryu JH, Kwon IC. Alliance with EPR effect: combined strategies to improve the epr effect in the tumor microenvironment. Theranostics 2019;9(26):8073-8090

92 Danhier F. To exploit the tumor microenvironment: since the EPR effect fails in the clinic, what is the future of nanomedicine. J Control Release 2016;244:108-121 